\title{
A SURVEY OF PROPER-MOTION STARS. XVI. ORBITAL SOLUTIONS FOR 171 SINGLE-LINED SPECTROSCOPIC BINARIES ${ }^{1}$
}

\author{
David W. Latham, Robert P. Stefanik, Guillermo Torres, and Robert J. Davis \\ Harvard-Smithsonian Center for Astrophysics, 60 Garden Street, Cambridge, MA 02138; dlatham@cfa.harvard.edu, \\ rstefanik@cfa.harvard.edu,gtorres@cfa.harvard.edu,rdavis@cfa.harvard.edu \\ TSEVI MAZEH \\ School of Physics and Astronomy, Raymond and Beverly Sackler Faculty of Exact Sciences, Tel Aviv University, Tel Aviv, Israel; \\ mazeh@wise7.tau.ac.il \\ BRUCE W. CARNEY \\ Department of Physics and Astronomy, University of North Carolina, Chapel Hill, NC 27599-3255; bruce@astro.unc.edu \\ JOHN B. LAIRD \\ Department of Physics and Astronomy, Bowling Green State University, Bowling Green, OH 43403; laird@tycho.bgsu.edu \\ AND \\ Jon A. Morse \\ Center for Astrophysics and Space Astronomy, Department of Astrophysical and Planetary Sciences, \\ Campus Box 389, University of Colorado, Boulder, CO 80309; morsey@casa.colorado.edu \\ Received 1999 May 4; accepted 2002 April 22
}

\begin{abstract}
We report 25,563 radial velocity measurements for 1359 single-lined stars in the Carney-Latham sample of 1464 stars selected for high proper motion. For 171 of these, we present spectroscopic orbital solutions. We find no obvious difference between the binary characteristics in the halo and the disk populations. The observed frequency is the same, and the period distributions are consistent with the hypothesis that the two sets of binaries were drawn from the same parent population. This suggests that metallicity in general, and radiative opacities in particular, have little influence over the fragmentation process that leads to short-period binaries. All the binaries with periods shorter than 10 days have nearly circular orbits, while the binaries with periods longer than 20 days exhibit a wide range of eccentricities and a median value of 0.37 . For the metalpoor high-velocity halo binaries in our sample, the transition from circular to eccentric orbits appears to occur at about 20 days, supporting the conclusion that tidal circularization on the main sequence is important for the oldest binaries in the Galaxy.
\end{abstract}

Key words: binaries: spectroscopic - Galaxy: halo

On-line material: machine-readable tables

\section{INTRODUCTION}

In 1981 May, we obtained our first echelle spectrum of the very metal-poor star G64-12. We wanted to test the feasibility of using our instrumentation for a major survey of metal-poor stars in the solar neighborhood to see what we could learn about the formation and early history of our Galaxy by studying the metallicities and kinematics of the halo and disk populations. The success of that first exposure convinced us to proceed with our survey (Carney \& Latham 1987) of a large sample of $G$ and $K$ dwarfs, selected for high proper motion in order to enhance the proportion of highvelocity metal-poor objects. Our kinematic selection proved to be effective, and more than a third of our stars have characteristics typical of the halo population, up by a large factor compared to the number density of about two per thousand for halo stars in the solar neighborhood.

When we undertook our survey, it was commonly believed that spectroscopic binaries were rare among the oldest populations of the Galaxy, such as the globular clusters and the high-velocity metal-poor field stars (cf. Jaschek

\footnotetext{
${ }^{1}$ Some of the results presented here used observations made with the Multiple Mirror Telescope, a joint facility of the Smithsonian Institution and the University of Arizona.
}

\& Jaschek 1957; Abt \& Levy 1969; Crampton \& Hartwick 1972; Gunn \& Griffin 1979). From the very beginning of the project, we planned to investigate the binaries in the halo versus the disk populations to see if we could learn why binaries were so rare in the halo. Thus, we have used the Center for Astrophysics (CfA) Digital Speedometers (Latham 1985, 1992) over the ensuing years to monitor the radial velocities of nearly all 1464 stars in our full sample (Carney et al. 1994, hereafter Paper XII), with the goal of deriving as many spectroscopic orbits as possible.

Preliminary orbital solutions for 80 of the binaries have already been published (Latham et al. 1988, hereafter Paper VI; Latham et al. 1992, hereafter Paper XI) in two batches of 40 . Even with just the first set of 40 orbits it was clear that there were many halo binaries, and that there was no obvious difference between the frequency and orbital characteristics of the binaries in the halo and disk populations (Paper VI; Torres 1991). This confirmed and very much strengthened a hint from the work of Stryker et al. (1985), who reported that spectroscopic binaries among Population II stars might be more common than previously thought.

For the second set of 40 orbits, the focus turned to the most metal-poor binaries in our sample, those with $[\mathrm{m} / \mathrm{H}]$ $<-1.6$. The goal was to investigate the role of tidal circularization in a (more or less) coeval sample of very old binaries 
and to estimate the orbital period corresponding to the transition from circular to eccentric orbits. The conclusion was that the transition period appeared to be about 20 days, considerably longer than the transition period of 12 days found for the solar-age open cluster M67, thus lending support to the interpretation that tidal circularization on the main sequence determines the transition period for populations of old binaries (Mathieu et al. 1992).

For our study of the transition period, we argued that it was not necessary to have orbital solutions for a complete sample of binaries, because we could not think of any selection effect that would bias our result. The situation is very different when the time comes to analyze the global distributions of the binary characteristics, such as the eccentricity and period distributions, or the secondary mass distribution. Here it is essential to understand and correct for incompletenesses. With the goal of reducing the size of the incompleteness corrections, we have continued to monitor all the stars identified as binaries but without orbital solutions. We have now reached the point of diminishing returns, and the rate of new orbital solutions has slowed to a trickle. The time has come to assemble all our orbital solutions in a homogeneous way and to proceed with the analysis of the binary characteristics.

During the 10 years since the publication of the second set of 40 orbits, we have refined our techniques for deriving radial velocities from our digital spectra. For the templates in our correlation analysis, we now use synthetic spectra calculated by Jon Morse for an extensive grid of model atmospheres computed using the ATLAS9 code developed by Kurucz (Morse \& Kurucz 2002). For the determination of the velocities of the individual stars in systems exhibiting composite spectra, we now use the two-dimensional correlation technique TODCOR (Zucker \& Mazeh 1994) for double-lined systems and an extension of TODCOR to three dimensions for triple-lined systems (Zucker, Torres, \& Mazeh 1995). These new techniques, together with new observations, have allowed us to improve the orbital determinations for the first 80 binaries. Moreover, as a result of the additional time coverage and richer data sets now available, we have been able to derive orbits for many additional systems.

In this paper, we report orbital solutions for 171 singlelined spectroscopic binaries. Preliminary CfA orbits were published for 27 of these in Paper VI and 27 in Paper XI. Updated orbits for those 54 binaries are included in this paper. Single-lined orbits have already been published for six of the 10 metal-poor field blue stragglers in our sample (Carney et al. 2001, hereafter Paper XIV) and are not repeated here. Double-lined orbits for 34 stars in our sample are reported by Goldberg et al. (2002, hereafter Paper XV). A single-lined orbit for a metal-poor red giant in our sample, $\mathrm{BD}+13^{\circ} 3683(=\mathrm{G} 141-19)$, is reported by Carney et al. (2002) and is not repeated here. Finally, we do not include an orbital solution for HD 114762, a member of our sample that is orbited by a candidate planetary companion (cf. Latham et al. 1989; Mazeh, Latham, \& Stefanik 1996; Marcy et al. 1999).

\section{RADIAL VELOCITIES}

The radial velocities of nearly all of the 1464 stars in our full sample (Paper XII) have been monitored with the CfA Digital Speedometers (Latham 1985, 1992). Three nearly identical instruments have been used on the Multiple Mirror Telescope and $1.5 \mathrm{~m}$ Tillinghast Reflector at the F. L. Whipple Observatory atop Mount Hopkins, Arizona, and on the $1.5 \mathrm{~m}$ Wyeth Reflector located at the Oak Ridge Observatory in the town of Harvard, Massachusetts. Echelle spectrographs have been used with photon counting intensified Reticon detectors to record about $45 \AA$ of spectrum in a single order. Most of the spectra were centered near $5187 \AA$, but a significant number of the early spectra were centered near $5197 \AA$. The shift in central wavelength was adopted in order to include all three lines of the $\mathrm{Mg} b$ triplet, after it was fully appreciated that all the other lines in our spectral window became extremely weak for our most metal-poor stars. The spectral resolution is about $8.5 \mathrm{~km} \mathrm{~s}^{-1}$ for all our exposures, and the signal-to-noise ratios range from about 5 to 50 per resolution element.

Radial velocities were extracted from the observed spectra using the one-dimensional correlation package R2RVSAO (Kurtz \& Mink 1998) running inside the IRAF ${ }^{2}$ environment. For the templates, we used a new grid of synthetic spectra (Morse \& Kurucz 2002) calculated using the latest Kurucz model atmospheres. The new grid of synthetic templates incorporates several improvements compared with the older grid that we used for several years (e.g., Nordström et al. 1994; Paper XV). Considerable effort was invested in the development of a revised line list for the wavelength region 5146-5229 $\AA$, with careful verification that the calculated spectra matched the high-quality observed spectra available for the sun (Kurucz et al. 1984). This extends the wavelength coverage of the old grid so that it now safely covers the entire range of observed wavelengths for any radial velocity likely to be encountered. In addition, the new grid extends the range of metallicities (typically $[\mathrm{m} / \mathrm{H}]=-4.0$ to +0.5 ) with uniform coverage over a much larger range of temperatures. With the goal of deriving more reliable radial and rotational velocities, especially for the slower rotations, the new grid of templates is more finely divided in rotational velocity, with typical values being $v_{\text {rot }}=0,1,2,4,6,8,10,12,16,20,25,30,35,40$, $50,60,70,80,90,100,120$, and $140 \mathrm{~km} \mathrm{~s}^{-1}$.

To select the optimum synthetic template for each star, we ran correlations for an appropriate range of template parameters and then adopted the template that gave the highest average peak correlation. Values for the effective temperature, $T_{\text {eff, }}$, and metallicity, $[\mathrm{m} / \mathrm{H}]$, were reported in Paper XII for about $85 \%$ of our stars, and in most cases the optimum template proved to have parameters very close to the values in Paper XII. Although it is reassuring that our procedure for selecting optimum templates picks stellar parameters that appear to be realistic in most cases, we must caution that our procedure was designed to optimize the radial velocity determinations, not to determine the fundamental astrophysical characteristics of our stars. In particular, we did not allow surface gravity to be a free parameter, but adopted $\log g=4.5$ for the stars with $T_{\text {eff }}<6000 \mathrm{~K}$ and $\log g=4.0$ for the hotter stars.

The template parameters adopted for the final velocity reductions of the 1359 stars reported in this paper are listed in Table 1. Throughout this paper, we use the star names

\footnotetext{
${ }^{2}$ IRAF is distributed by the National Optical Astronomy Observatory, which is operated by the Association of Universities for Research in Astronomy, Inc., under contract with the National Science Foundation.
} 
TABLE 1

Adopted Template Parameters

\begin{tabular}{|c|c|c|c|c|c|c|c|}
\hline Star & R.A. (J2000.0) & Decl. (J2000.0) & $\begin{array}{l}T_{\text {eff }} \\
(\mathrm{K})\end{array}$ & {$[\mathrm{m} / \mathrm{H}]$} & $\begin{array}{c}v_{\text {rot }} \\
\left(\mathrm{km} \mathrm{s}^{-1}\right)\end{array}$ & $\begin{array}{c}\log g \\
\left(\mathrm{~cm} \mathrm{~s}^{-2}\right)\end{array}$ & Paper XII \\
\hline G130-32 ...... & 000004.0 & +341118 & 5750 & 0.0 & 4 & 4.5 & G130-32 \\
\hline G158-21 ...... & 000058.4 & -045557 & 5500 & -0.5 & 2 & 4.5 & G158-21 \\
\hline G217-29...... & 000303.4 & +564359 & 5250 & 0.5 & 2 & 4.5 & G217-29 \\
\hline G30-39........ & 000421.5 & +125726 & 5500 & 0.0 & 0 & 4.5 & G30-39 \\
\hline G217-30 ...... & 000426.9 & +580407 & 5000 & -0.5 & 0 & 4.5 & $\mathrm{G} 217-30$ \\
\hline
\end{tabular}

Note.-Table 1 is presented in its entirety in the electronic edition of the Astronomical Journal. A portion is shown here for guidance regarding its form and content.

exactly as they appear in our observing catalogs to facilitate the bookkeeping. The observing catalog names are mostly based on the Giclas identifications (Giclas, Burnham, \& Thomas 1971, 1978), but a few are the Henry Draper or $\mathrm{SAO}$ catalog numbers ( $\mathrm{H}$ and $\mathrm{S}$ prefixes, respectively). Some of the star names used in Paper XII differ from our observing catalogs, and B1950.0 coordinates were used in Paper XII instead of J2000.0, so we have provided the names from Paper XII in the final column of Table 1 to assist users in matching stars with entries in Paper XII.

The individual Heliocentric Julian Dates, heliocentric radial velocities, and velocity error estimates from R2RVSAO are listed for 25,563 observations of 1359 stars in Table 2. These velocities are given in kilometers per second on the native CfA system as defined by nightly observations of the dawn and dusk sky. To convert to an absolute velocity system based on extensive observations of minor planets, $0.139 \mathrm{~km} \mathrm{~s}^{-1}$ should be added to the native CfA velocities (Stefanik, Latham, \& Torres 1999; that paper states incorrectly that $0.139 \mathrm{~km} \mathrm{~s}^{-1}$ should be subtracted from the CfA native velocities to convert to absolute velocities). The first column in Table 2 gives a code for the telescope used.

One of the advantages of our new grid of synthetic templates is that the wavelength coverage, 5146.02-5229.21 A, is considerably wider than the wavelength window of our observed spectra, normally $5165.77-5211.23 \AA$. This provides more than $1000 \mathrm{~km} \mathrm{~s}^{-1}$ of leeway on both ends of our template spectra and allows us to use the entire observed spectrum in our correlation analysis, no matter how large the Doppler shift (the largest known radial velocity for a

TABLE 2

Radial Velocities

\begin{tabular}{|c|c|c|c|}
\hline Tel & HJD & $v_{\text {rad }}$ & $\sigma$ \\
\hline \multicolumn{4}{|c|}{ G130-32: $00^{\mathrm{h}} 00^{\mathrm{m}} 04^{\mathrm{s}} \cdot 0,+34^{\circ} 11^{\prime} 18^{\prime \prime}$} \\
\hline $\mathrm{T} \ldots \ldots \ldots \ldots \ldots \ldots$ & $2,445,247.6669$ & -27.62 & 0.41 \\
\hline \multirow{4}{*}{$\mathrm{W} \ldots \ldots \ldots \ldots \ldots$} & $2,445,570.8111$ & -32.42 & 0.39 \\
\hline & $2,445,587.7710$ & -33.42 & 0.76 \\
\hline & $2,445,917.8699$ & -29.71 & 0.72 \\
\hline & $2,445,920.8867$ & -31.09 & 0.70 \\
\hline
\end{tabular}

Notes.-The telescopes are as follows: (W) Wyeth Reflector (12,200 observations), (T) Tillinghast Reflector (10,094 observations), and (M) Multiple Mirror Telescope (3269 observations). Table 2 is presented in its entirety in the electronic edition of the Astronomical Journal. A portion is shown here for guidance regarding its form and content. star in our Galaxy is $-583.7 \mathrm{~km} \mathrm{~s}^{-1}$ for G233-27). When an observed template is used and the wavelength window is the same as for the observed object spectrum, then the correlation analysis loses spectral coverage at both ends of the wavelength window by the amount of the relative Doppler shift between the template star and the object, including the changing Doppler shift due to the earth's motion. For highvelocity stars, this loss of spectral information can be very significant for the CfA Digital Speedometers, because the wavelength window is only $45.46 \AA$. In the case of G233-27, the loss of spectral coverage would be almost $50 \%$ !

R2RVSAO provides the option of iterating the correlation analysis. In subsequent iterations the velocity from the previous iteration is used to pick out the section of the template spectrum that matches the rest-wavelength window of the observed spectrum, so that all of the observed spectrum is used in the final analysis. This iteration converges quickly, and two passes (NZPASS $=2$ ) are sufficient to achieve an accurate match of the template and object rest-wavelength windows.

One of the concerns raised by the use of a small wavelength window in a correlation analysis is that not very many spectral lines are included in the window. This increases the risk of systematic errors due to effects such as a mismatch in the spectral type of the template compared with the object, or the shifting of key lines off one end of the spectrum or the other. To illustrate the impact of adjusting the wavelength window to get an exact match between the template and object spectra, we plot in Figure 1 the difference in

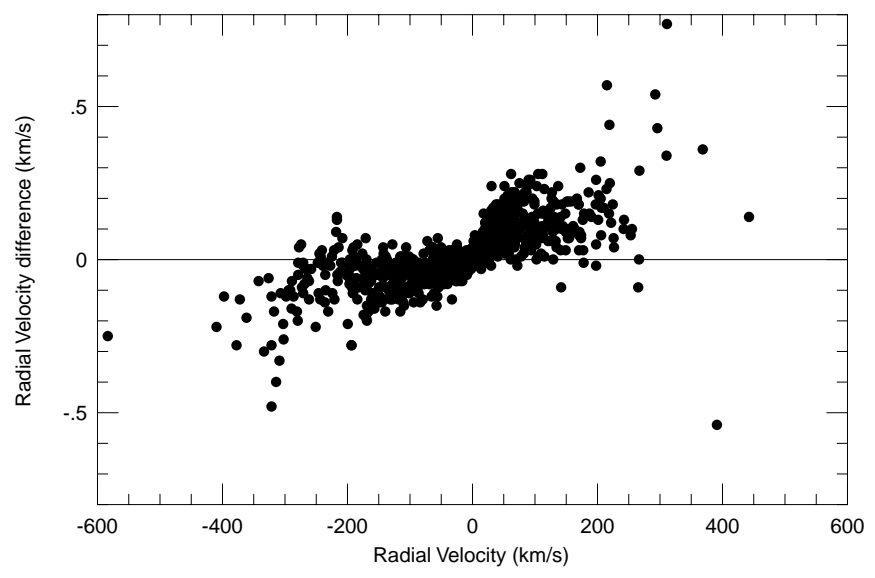

FIG. 1.-Differences in the mean velocities for 1359 stars using two iterations to shift the template window to match the observed window vs. no shift. 
the mean velocities for all 1359 of our stars, namely the mean velocity calculated with two iterations in the template shift compared with no shift. For small velocities, the difference is small at the $0.1 \mathrm{~km} \mathrm{~s}^{-1}$ level. However, for stars with extreme velocities, the difference can be as large as $0.5 \mathrm{~km}$ $\mathrm{s}^{-1}$. Moreover, there is a systematic trend, with the difference being negative for blueshifted velocities and positive for redshifted velocities. This can introduce a significant systematic error (e.g., $1 \%$ ) in the orbital amplitude derived for spectroscopic binaries if the template and object wavelength windows are not matched.

For the velocities reported in this paper, we used two iterations (NZPASS $=2$ ) to match the wavelength windows of the template and object spectra. In all our previously published results from the CfA Digital Speedometers, no iteration was used.

\section{IDENTIFICATION OF SPECTROSCOPIC BINARIES}

In this section, we describe the procedures that we now use for the identification and follow-up of spectroscopic binaries. Although these procedures have evolved in detail, the overall strategy has remained the same throughout this project.

The CfA Digital Speedometers are normally scheduled for telescope time during the bright of moon. This has led to a natural cycle of a monthly review of those stars with new observations and the preparation of a revised observing plan for the next month. As soon as a star was suspected to show variable velocity, we increased its observing priority, with the goal of adding a new velocity each month and more frequently for stars that were suspected to have very short periods.

When only a few velocity observations are available, the first indication that a star is a spectroscopic binary usually comes from the rms velocity deviations being larger than expected and/or from a noticeable pattern in the history of the velocities, such as a drift with time. We have found that the $\chi^{2}$ probability, $P\left(\chi^{2}\right)$ (e.g., Press et al. 1992), is useful for identifying candidate variables, especially when the velocity errors cover a range of values, depending on a variety of factors such as the rotational velocity, metallicity, and effective temperature (not to mention exposure level). In this situation, it is essential to have a reliable estimate for the individual uncertainty, $\sigma_{i, \text { int }}$, in each velocity determination when calculating $\chi^{2}$ :

$$
\chi^{2}=\sum_{i=1}^{n}\left(\frac{x_{i}-\left\langle x_{i}\right\rangle}{\sigma_{i, \mathrm{int}}}\right)^{2} .
$$

$P\left(\chi^{2}\right)$ gives the probability that the observed $\chi^{2}$ value or larger could result by accident for observations of a constant star with Gaussian errors. For a large sample of constant stars, the distribution of $P\left(\chi^{2}\right)$ values should be flat. The observed distribution of $P\left(\chi^{2}\right)$ values for all 1359 stars is shown in Figure 2. Except for the strong peak of a few hundred stars in the first bin, which includes all our binary stars, the distribution is flat as expected. However, to get the histogram to come out flat, we found it necessary to include a floor error contribution of $0.25 \mathrm{~km} \mathrm{~s}^{-1}$, combined in quadrature with the individual error estimates from R2RVSAO. Without this floor error, the histogram has a mild slope up toward smaller values of $P\left(\chi^{2}\right)$ in the range $0.1-1.0$. For larger floor errors, the histogram slopes up toward higher
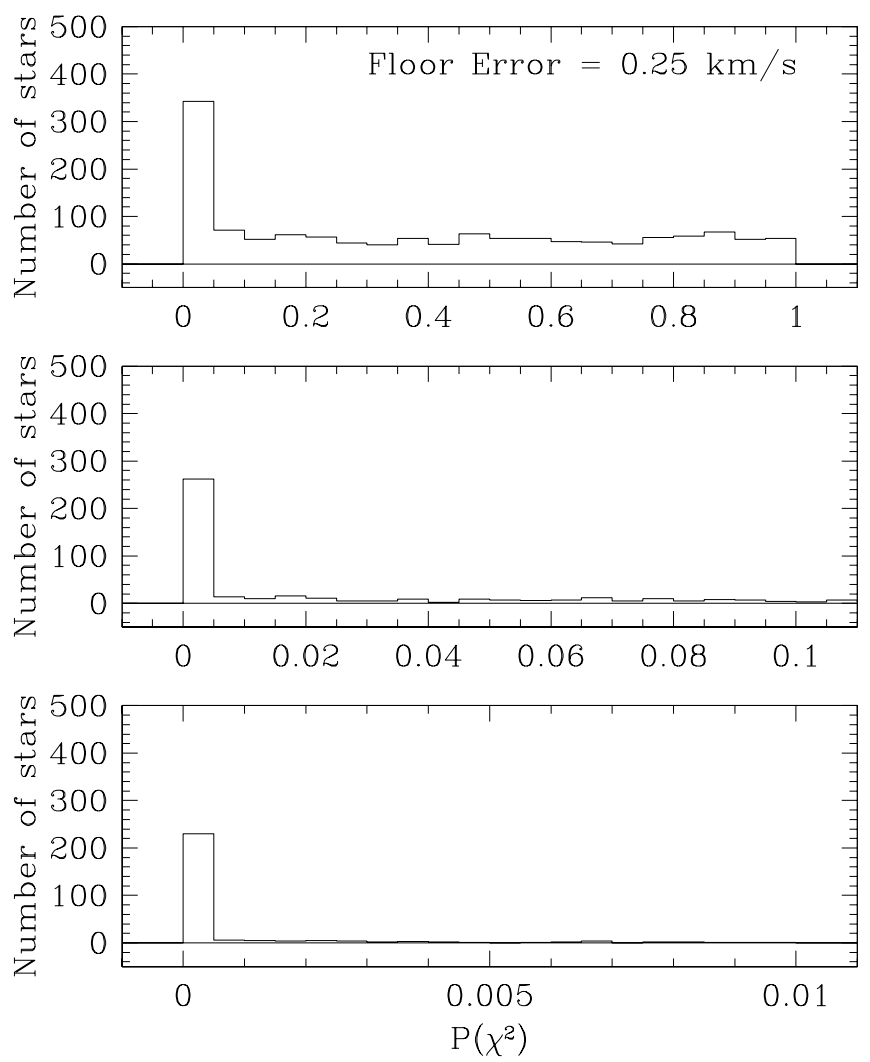

FIG. 2.-Histograms for the $\chi^{2}$ probability, $P\left(\chi^{2}\right)$. The bottom panels focus on regions of small values for $P\left(\chi^{2}\right)$.

values of $P\left(\chi^{2}\right)$. The internal error estimates provided by R2RVSAO do not include the contribution of systematic errors, such as residual errors that remain in the nightly velocity zero points after the monthly run corrections have been applied. The inclusion of a floor error appears to account for such systematic errors.

The bottom two panels in Figure 2 show that most of the stars at the peak in the first bin actually have very small values of $P\left(\chi^{2}\right)$, less than 0.001 . It is our experience that by the time we have accumulated enough velocity observations to solve for a reliable spectroscopic orbit, the $P\left(\chi^{2}\right)$ is much smaller than 0.001 . However, when only a few observations are available, the $P\left(\chi^{2}\right)$ value for a binary can sometimes avoid being small, either by accident or because the orbit has a long period and/or low amplitude and/or high eccentricity and has not yet revealed itself. Therefore, we give special attention to follow-up observations of stars with $P\left(\chi^{2}\right)$ values smaller than about 0.01 .

Some of the stars with very small $P\left(\chi^{2}\right)$ values do not appear to be spectroscopic binaries, in the sense that we cannot find a plausible orbital solution for them. Many of these are bright stars with strong exposures and sharp lines, and therefore they have unusually small estimated velocity errors. $P\left(\chi^{2}\right)$ assumes that the errors are Gaussian, but real data sets always seem to have more outliers. We suspect that this is the source of some of the small values of $P\left(\chi^{2}\right)$.

As part of our monthly reviews, we normally inspect plots of the velocity history of each star that has new observations. Slow velocity drifts are sometimes apparent on these plots before enough of a trend has been covered to bring the $P\left(\chi^{2}\right)$ down to a small value. We then monitor the velocities of such stars with a cadence appropriate to the drift pattern. 
For the many stars that showed no obvious signature of velocity variation in our monthly reviews, we followed an observing strategy designed to accumulate 10 observations over a total time span of at least 3000 days or until a robust orbital solution emerged. Figure 3 shows histograms for the number of observations and time span for all 1359 stars. Most of the spectroscopic binaries have more than 20 observations, and some of the binaries with short periods were observed for a time span of less than 3000 days. Otherwise, we came close to our observing goals, with more than seven observations and 3000 days of coverage for nearly all our targets.

The velocity results for all 1359 stars are summarized in Table 3, where we present the number of observations, $N_{\text {obs; }}$; the time span of the observations in days; the projected rotational velocity, $v_{\text {rot }}$ in kilometers per second, derived using a quadratic interpolation for the template giving the highest average peak correlation value plus one template on either side; the mean heliocentric radial velocity, $v_{\text {rad }}$, in kilometers per second on the native CfA system $\left(0.139 \mathrm{~km} \mathrm{~s}^{-1}\right.$ should be added to these velocities to put them on the absolute velocity system defined by our observations of minor planets); the standard deviation of the mean velocity, $\sigma$; the external rms of the deviations from the mean, ext; the mean of the internal error estimates from R2RVSAO combined in quadrature with a floor error of $0.25 \mathrm{~km} \mathrm{~s}^{-1}$, int; the ratio of external to internal error, $e / i ; \chi^{2}$; and the $\chi^{2}$ probability, $P\left(\chi^{2}\right)$. The penultimate column reports our assignment of targets to the halo, H, or disk, D, using the Galactic $V$ velocity and metallicity, as illustrated in Figure 7. When either the Galactic $V$ velocity or metallicity is unavailable, this column is blank. The final column indicates the binaries we have identified, with "SO" for the binaries with robust orbits reported in Table 4, "SP" for the binaries with preliminary orbits reported in Table 5, and " $S$ " for definite binaries lacking period determinations, those shown in Figure 6 . Note that for the binaries with orbital solutions, the systemic velocities and uncertainties reported in Tables 4 and 5 are more appropriate for most applications than the mean velocities and uncertainties reported in Table 3 .

For some binaries, the secondary star is bright enough so that its spectrum can also be detected. Double-lined systems were often recognized after only a few exposures had been obtained, because the one-dimensional correlation plots showed obvious doubling of the peaks. However, several of the systems with composite spectra were less obvious, because either the secondary was much fainter than the primary or the orbital amplitude was so small that the two cor-
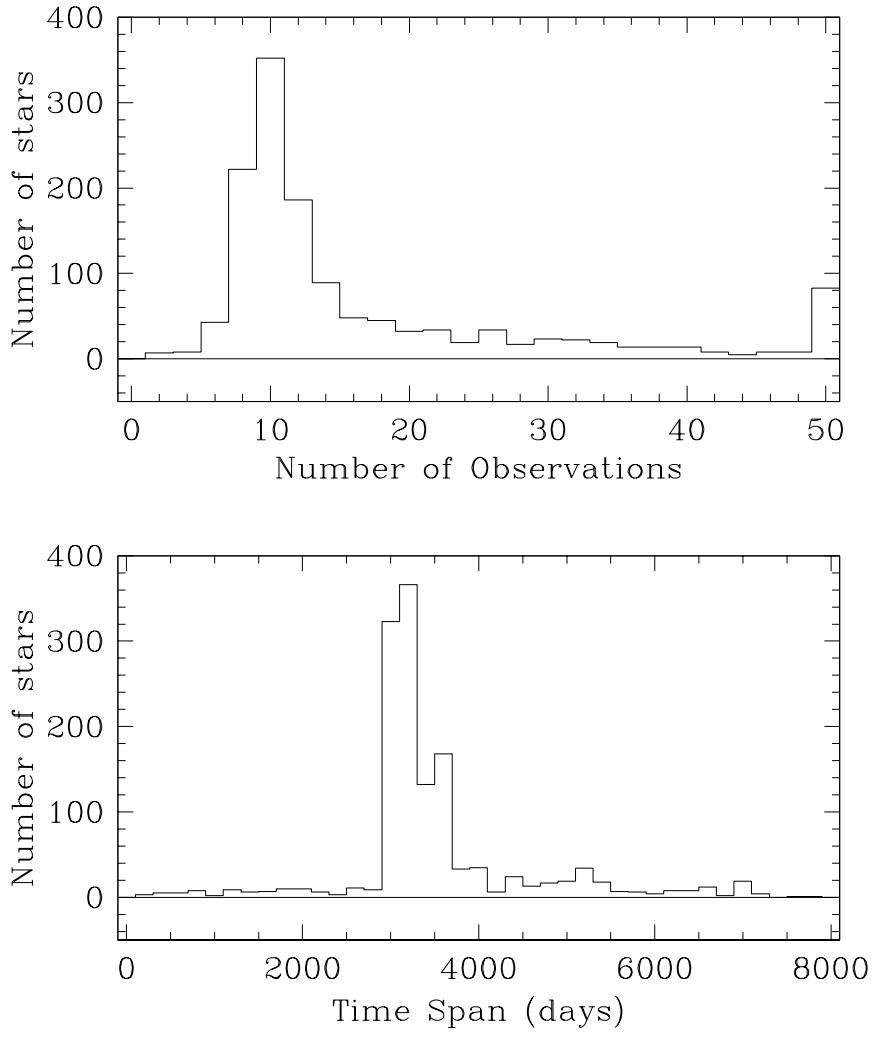

FIG. 3.-Histograms for the number of observations and time spanned.

relation peaks were always blended or the secondary peak stayed hidden by the primary peak for a long time because the period was long and/or the eccentricity was high.

A few of our binaries were first suspected to be doublelined only after we had derived preliminary orbital solutions, and then we could see that the velocity residuals had a larger scatter than expected and/or the minimum mass for the secondary was nearly as large as the primary mass and/ or the velocities near the center-of-mass velocity showed systematic deviations due to blending between the primary and secondary correlation peaks. In these cases, the preliminary orbital solution for the primary could be used as a guide for predicting where to look for the secondary spectrum.

A few of our binaries were first recognized to have composite spectra because the metallicity determinations

TABLE 3

Mean Velocities and ERrors

\begin{tabular}{|c|c|c|c|c|c|c|c|c|c|c|c|c|c|c|}
\hline Star & $\begin{array}{c}\text { R.A. } \\
(\mathrm{J} 2000.0)\end{array}$ & $\begin{array}{c}\text { Decl. } \\
(\mathrm{J} 2000.0)\end{array}$ & $N_{\text {obs }}$ & Span & $v_{\text {rot }}$ & $v_{\text {rad }}$ & $\sigma$ & ext & int & $\mathrm{e} / \mathrm{i}$ & $\chi^{2}$ & $P\left(\chi^{2}\right)$ & Disk/Halo & $\begin{array}{c}\text { Binary } \\
\text { Type }\end{array}$ \\
\hline G130-32 ...... & 000004.0 & +341118 & 46 & 1886 & 3 & -31.18 & 0.28 & 1.92 & 0.53 & 3.60 & 698.00 & 0.000000 & D & SO \\
\hline G217-29...... & 000303.4 & +564359 & 10 & 3308 & 3 & -50.45 & 0.21 & 0.67 & 0.52 & 1.28 & 13.21 & 0.153388 & & \\
\hline G30-39........ & 000421.5 & +125726 & 10 & 3328 & 0 & 18.22 & 0.19 & 0.61 & 0.54 & 1.13 & 12.90 & 0.167097 & D & \\
\hline G217-30 ..... & 000426.9 & +580407 & 18 & 3275 & 0 & -90.15 & 0.15 & 0.54 & 0.63 & 0.86 & 16.14 & 0.513665 & $\mathrm{D}$ & \\
\hline
\end{tabular}

Note.-Table 3 is presented in its entirety in the electronic edition of the Astronomical Journal. A portion is shown here for guidance regarding its form and content. 
TABLE 4

156 Robust Orbital Solutions

\begin{tabular}{|c|c|c|c|c|c|c|c|c|c|c|c|c|}
\hline Star & $\begin{array}{c}\text { R.A. } \\
(\mathrm{J} 2000.0)\end{array}$ & $\begin{array}{c}\text { Decl. } \\
(\mathrm{J} 2000.0)\end{array}$ & $P$ & $\gamma$ & $K$ & $e$ & $\omega$ & $T$ & $a_{A} \sin i$ & $f(m)$ & $\begin{array}{c}N, \\
\sigma\end{array}$ & $\begin{array}{l}\text { Span } \\
\text { Cycles }\end{array}$ \\
\hline G130-32 ..... & 000004.0 & +341118 & $\begin{array}{r}953 \\
\pm 14\end{array}$ & $\begin{array}{r}-30.639 \\
\pm 0.093\end{array}$ & $\begin{array}{r}3.22 \\
\pm 0.18\end{array}$ & $\begin{array}{r}0.470 \\
\pm 0.042\end{array}$ & $\begin{array}{l}44.5 \\
\pm 6.6\end{array}$ & $\begin{array}{r}46303 \\
\pm 12\end{array}$ & $\begin{array}{r}37.2 \\
\pm 2.0\end{array}$ & $\begin{array}{r}0.00226 \\
\pm 0.00034\end{array}$ & $\begin{array}{l}46 \\
0.54\end{array}$ & $\begin{array}{r}1886.0 \\
2.0\end{array}$ \\
\hline G265-1 ........ & 000850.8 & +864716 & $\begin{array}{l}71.55 \\
\pm 0.17\end{array}$ & $\begin{array}{r}-79.71 \\
\pm 0.17\end{array}$ & $\begin{array}{r}13.55 \\
\pm 0.23\end{array}$ & $\begin{array}{r}0.221 \\
\pm 0.015\end{array}$ & $\begin{array}{r}140.8 \\
\pm 4.6\end{array}$ & $\begin{array}{r}46405.92 \\
\pm 0.83\end{array}$ & $\begin{array}{r}13.00 \\
\pm 0.30\end{array}$ & $\begin{array}{r}0.0171 \\
\pm 0.0012\end{array}$ & $\begin{array}{l}26 \\
0.71\end{array}$ & $\begin{array}{r}658.0 \\
9.2\end{array}$ \\
\hline G171-58 ..... & 002508.7 & +480251 & $\begin{array}{r}355.5 \\
\pm 1.1\end{array}$ & $\begin{array}{l}-0.03 \\
\pm 0.15\end{array}$ & $\begin{array}{r}2.93 \\
\pm 0.19\end{array}$ & $\begin{array}{r}0.306 \\
\pm 0.063\end{array}$ & $\begin{array}{r}41 \\
\pm 13\end{array}$ & $\begin{array}{r}47200 \\
\pm 10\end{array}$ & $\begin{array}{l}13.6 \\
\pm 1.1\end{array}$ & $\begin{array}{r}0.00080 \\
\pm 0.00019\end{array}$ & $\begin{array}{l}38 \\
0.68\end{array}$ & $\begin{array}{r}4517.6 \\
12.7\end{array}$ \\
\hline G1-21A....... & 004518.2 & +014031 & $\begin{array}{r}2037 \\
\pm 15\end{array}$ & $\begin{array}{r}-37.94 \\
\pm 0.16\end{array}$ & $\begin{array}{r}5.05 \\
\pm 0.63\end{array}$ & $\begin{array}{r}0.464 \\
\pm 0.075\end{array}$ & $\begin{array}{r}355.5 \\
\pm 5.6\end{array}$ & $\begin{array}{r}48293 \\
\pm 25\end{array}$ & $\begin{array}{l}125 \\
\pm 17\end{array}$ & $\begin{array}{r}0.0188 \\
\pm 0.0077\end{array}$ & $\begin{array}{l}33 \\
0.77\end{array}$ & $\begin{array}{r}4085.8 \\
2.0\end{array}$ \\
\hline
\end{tabular}

Note.-Table 4 is presented in its entirety in the electronic edition of the Astronomical Journal. A portion is shown here for guidance regarding its form and content.

showed a variation with the same period as the orbit (Paper XII). The metallicity was the highest when the two stars passed through the center-of-mass velocity, while the metallicity was lower for the orbital phases when the lines of one star tended to get filled in by the continuum of the other star.

These procedures led to the identification of 47 stars with composite spectra, three of them triple-lined. Double-lined orbits using TODCOR velocities are reported for 34 of these systems in Paper XV. That paper does not include three double-lined binaries in our sample with very short periods and rapid rotation: G71-3, G136-99, and G24-18P. G24$18 \mathrm{P}$ is especially interesting. It is a metal-poor, double-lined eclipsing binary for which the metallicity can be inferred, despite its very rapid rotation, because the nearby propermotion companion, G24-18F, is a sharp-lined single star. A careful analysis of this system is underway.

As part of the final review of the 25,563 observations of 1359 stars included in this paper, we inspected plots of each observed spectrum and the corresponding one-dimensional correlation plots to look for previously missed double-lined binaries, among other things. Three additional double-lined systems with very faint secondaries were identified in this review: G71-33, G72-18, and G161-84. Single-lined velocities and orbital solutions for these three stars are included in this paper.
We have identified 11 spectroscopic triple systems in our sample, belonging to three main types:

Triple-lined spectra.-G103-50 and G87-45 both consist of an inner double-lined binary with an orbital solution and an outer companion with period too long to yield an orbital solution yet.

Double-lined spectra.-G161-82, G176-27, and G262-22 each consist of an inner double-lined binary with an orbital solution and an unseen outer companion with a solution for its orbit around the center of mass of the inner binary. G176-46 (Latham et al. 1992) and G184-23 also consist of a double-lined inner binary with an orbital solution, but for these two systems the period of the unseen outer companion is too long to yield an orbital solution yet.

Single-lined spectra.-G30-52, G38-13 (Mazeh, Krymolowski, \& Latham 1993), G111-33, and G25-15 each consist of a inner single-lined binary with an orbital solution and an unseen outer companion also with an orbital solution.

For these triples, the full details of the orbital solutions have been published only for G38-13. We do plan to publish additional papers for the other triple systems and therefore do not include any of them in this paper. The rest of the 1464 stars from Paper XII that are not included among the 1359 stars reported here all fall in the following categories:

TABLE 5

15 Preliminary Orbital Solutions

\begin{tabular}{|c|c|c|c|c|c|c|c|c|c|c|c|c|}
\hline Star & $\begin{array}{c}\text { R.A. } \\
(\mathrm{J} 2000.0)\end{array}$ & $\begin{array}{c}\text { Decl. } \\
(\mathrm{J} 2000.0)\end{array}$ & $P$ & $\gamma$ & K & $e$ & $\omega$ & $T$ & $a_{A} \sin i$ & $f(m)$ & $N, \sigma$ & $\begin{array}{c}\text { Span } \\
\text { Cycles }\end{array}$ \\
\hline $270-174 \ldots \ldots$. & 011318.8 & -015143 & $\begin{array}{r}14520 \\
\pm 3933\end{array}$ & $\begin{array}{l}-5.92 \\
\pm 0.16\end{array}$ & $\begin{array}{r}4.84 \\
\pm 0.30\end{array}$ & $\begin{array}{r}0.841 \\
\pm 0.049\end{array}$ & $\begin{array}{r}108.3 \\
\pm 5.5\end{array}$ & $\begin{array}{r}48851 \\
\pm 60\end{array}$ & $\begin{array}{r}523 \\
\pm 111\end{array}$ & $\begin{array}{r}0.0271 \\
\pm 0.0044\end{array}$ & $\begin{array}{l}71 \\
0.66\end{array}$ & $\begin{array}{r}6265.9 \\
0.4\end{array}$ \\
\hline G71-33 ....... & 014513.8 & +033049 & $\begin{array}{r}6583 \\
\pm 448\end{array}$ & $\begin{array}{r}-11.8 \\
\pm 1.1\end{array}$ & $\begin{array}{r}25 \\
\pm 94\end{array}$ & $\begin{array}{r}0.93 \\
\pm 0.24\end{array}$ & $\begin{array}{r}156 \\
\pm 47\end{array}$ & $\begin{array}{r}49414 \\
\pm 55\end{array}$ & $\begin{array}{r}840 \\
\pm 3103\end{array}$ & $\begin{array}{r}0.5 \\
\pm 6.1\end{array}$ & $\begin{array}{l}57 \\
0.88\end{array}$ & $\begin{array}{r}6683.8 \\
1.0\end{array}$ \\
\hline G36-33 ........ & 024809.1 & +270407 & $\begin{array}{r}6127 \\
\pm 799\end{array}$ & $\begin{array}{l}+9.34 \\
\pm 0.21\end{array}$ & $\begin{array}{r}2.82 \\
\pm 0.25\end{array}$ & $\begin{array}{r}0.686 \\
\pm 0.052\end{array}$ & $\begin{array}{r}116.5 \\
\pm 8.0\end{array}$ & $\begin{array}{r}48064 \\
\pm 69\end{array}$ & $\begin{array}{r}173 \\
\pm 30\end{array}$ & $\begin{array}{r}0.0055 \\
\pm 0.0014\end{array}$ & $\begin{array}{l}77 \\
0.58\end{array}$ & $\begin{array}{r}5176.9 \\
0.8\end{array}$ \\
\hline G102-20 ...... & 054009.7 & +121041 & $\begin{array}{r}9467 \\
\pm 1730\end{array}$ & $\begin{array}{r}+22.75 \\
\pm 0.59\end{array}$ & $\begin{array}{r}4.40 \\
\pm 0.29\end{array}$ & $\begin{array}{r}0.414 \\
\pm 0.047\end{array}$ & $\begin{array}{r}62 \\
\pm 12\end{array}$ & $\begin{array}{r}50747 \\
\pm 155\end{array}$ & $\begin{array}{r}522 \\
\pm 179\end{array}$ & $\begin{array}{r}0.063 \\
\pm 0.031\end{array}$ & & $\begin{array}{r}7099.5 \\
0.7\end{array}$ \\
\hline G115-52 ..... & 090650.5 & +470651 & $\begin{array}{r}5614 \\
\pm 447\end{array}$ & $\begin{array}{l}+7.414 \\
\pm 0.095\end{array}$ & $\begin{array}{r}8.08 \\
\pm 0.51\end{array}$ & $\begin{array}{r}0.940 \\
\pm 0.010\end{array}$ & $\begin{array}{r}85.9 \\
\pm 3.0\end{array}$ & $\begin{array}{r}49728.3 \\
\pm 1.8\end{array}$ & $\begin{array}{r}213 \\
\pm 14\end{array}$ & $\begin{array}{r}0.0122 \\
\pm 0.0015\end{array}$ & $\begin{array}{l}51 \\
0.63\end{array}$ & $\begin{array}{r}5214.0 \\
0.9\end{array}$ \\
\hline
\end{tabular}

Note.-Table 5 is presented in its entirety in the electronic edition of the Astronomical Journal. A portion is shown here for guidance regarding its form and content. 
stars with composite spectra (see above), white dwarfs, blue stragglers (Paper XIV), giants (Carney et al. 2002), a $B-V$ color redder than 1.00, HD 114762 (cf. Latham et al. 1989; Mazeh et al. 1996; Marcy et al. 1999), and four stars not observed with the CfA Digital Speedometers.

\section{ORBITAL SOLUTIONS}

As part of our monthly reviews, we analyzed promising binary candidates for possible orbital solutions, using the power spectrum of the velocities (e.g., Mazeh et al. 1993) to search for candidate periods. For our initial attempts at orbital solutions, we used a code based on ORB18 (Mazeh et al. 1993). This code is effective even when no candidate period has been identified, because it can be used to search for possible orbits at thousands of trial periods in a fine grid.

When a binary first gave an orbital solution that looked plausible, we normally continued to obtain additional observations of that star, often doubling the number of data points before deciding that the orbit was reliable. Only when the orbital solution was fully confirmed by additional observations and the velocity curve was well covered with observed points did we consider an orbital solution robust enough to stop observing the binary.

The details for 156 robust orbital solutions are presented in Table 4 and are illustrated in Figure 4, where we plot the individual observed velocities as a function of orbital phase together with the velocity curves calculated from the orbital solutions. The center-of-mass velocity, $\gamma$, is shown as a horizontal dashed line, and the orbital period is given at the end of each top label. In Table 4, we give the period $P$ in days, the center-of-mass velocity $\gamma$ in kilometers per second, the observed orbital semiamplitude $K$ in kilometers per second, the eccentricity $e$, the longitude of periastron $\omega$ in degrees, the Heliocentric Julian Date of periastron passage $T-2,400,000$, the projected semimajor axis $a_{A} \sin i$ in gigameters $(\mathrm{Gm})$, the mass function $f(\mathscr{M})$ in solar masses, the number of observations $N$, the rms velocity residuals $\sigma$, and the time span both in days and in the number of periods covered. The second line for each binary gives the uncertainties, except for the final two columns.

\section{NOTES TO TABLE 4}

For the systems resolved as visual binaries by Hipparcos, we list the separation (sep), position angle, and magnitude difference. For the unresolved systems that show curvature in the Hipparcos proper motions, we list the astrometric accelerations $\left(g_{\alpha}\right.$ and $\left.g_{\delta}\right)$ and the cosmic scatter $(\epsilon)$ for the systems that show excess unsolved scatter.

G171-58 $\left(00^{\mathrm{h}} 25^{\mathrm{m}} 08^{\mathrm{s}} .7,+48^{\circ} 02^{\prime} 51^{\prime \prime} ;\right.$ HIP 1987): visual companion, P.A. $=242^{\circ}$, sep $=0.277, \Delta H_{p}=2.91 \mathrm{mag}$; this cannot be the spectroscopic companion.

G32-56 $\left(00^{\mathrm{h}} 53^{\mathrm{m}} 14^{\mathrm{s}} .1,+19^{\circ} 08^{\prime} 48^{\prime \prime} ;\right.$ HIP 4166$): g_{\alpha}=-6.9$, $g_{\delta}=+4.7$ mas yr $^{-2}$.

G34-16 $\left(01^{\mathrm{h}} 16^{\mathrm{m}} 36^{\mathrm{s}} \cdot 4,+18^{\circ} 31^{\prime} 21^{\prime \prime}\right.$; HIP 5952$)$ : visual companion, P.A. $=264^{\circ}$, sep $=0.621, \Delta H_{p}=3.59 \mathrm{mag}$; this cannot be the spectroscopic companion.

G172-61 $\left(01^{\mathrm{h}} 34^{\mathrm{m}} 22^{\mathrm{s}} \cdot 3,+48^{\circ} 44^{\prime} 25^{\prime \prime} ;\right.$ ADS 1221A, IDS $01282+4814 \mathrm{~A})$.

G72-58 $\left(02^{\mathrm{h}} 08^{\mathrm{m}} 23^{\mathrm{s}} .8,+28^{\circ} 18^{\prime} 38^{\prime \prime} ;\right.$ HIP 9971$)$ : CPM with G72-59.
G72-59 $\left(02^{\mathrm{h}} 08^{\mathrm{m}} 23^{\mathrm{s}} \cdot 8,+28^{\circ} 18^{\prime} 17^{\prime \prime}\right.$; HIP 9972$)$ : CPM with G72-58.

G73-57 $\left(02^{\mathrm{h}} 26^{\mathrm{m}} 01^{\mathrm{s}} .7,+05^{\circ} 46^{\prime} 47^{\prime \prime}\right.$; HIP 11349): $g_{\alpha}=$

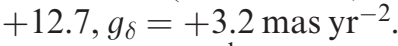

G173-59 (02 $33^{\mathrm{m}} 53^{\mathrm{s}} .7,+49^{\circ} 30^{\prime} 22^{\prime \prime} ;$ HIP 11923): $g_{\alpha}=$ $-12.7, g_{\delta}=+0.1$ mas $^{-2}{ }^{-2}$.

G220-13 $\left(02^{\mathrm{h}} 35^{\mathrm{m}} 32^{\mathrm{s}} .7,+59^{\circ} 47^{\prime} 32^{\prime \prime}\right.$; HIP 12062): $\epsilon=4.5$ mas.

G36-33 $\left(02^{\mathrm{h}} 48^{\mathrm{m}} 09^{\mathrm{s}} .1,+27^{\circ} 04^{\prime} 07^{\prime \prime}\right.$; HIP 13081): $g_{\alpha}=$ $+18.5, g_{\delta}=+3.4$ mas $\mathrm{yr}^{-2}$; Tokovinin (1992) noted that this was a spectroscopic binary with period longer than 3000 days.

G5-27 $\left(03^{\mathrm{h}} 18^{\mathrm{m}} 27^{\mathrm{s}} .1,+15^{\circ} 10^{\prime} 38^{\prime \prime} ;\right.$ HIP 15394): $g_{\alpha}=$ $+28.5, g_{\delta}=+6.8$ mas $^{-2}$.

G6-20 $\left(03^{\mathrm{h}} 37^{\mathrm{m}} 11^{\mathrm{s}} .0,+25^{\circ} 59^{\prime} 27^{\prime \prime}\right.$; HIP 16879): Photometric variable, period $=24.53$ days, amplitude $=0.05$ mag; a double-lined orbit has been published by Stockton \& Fekel (1992).

G95-57B $\left(03^{\mathrm{h}} 47^{\mathrm{m}} 02^{\mathrm{s}} .7,+41^{\circ} 25^{\prime} 40^{\prime \prime}\right.$; ADS 2757B): An orbit was published by Tokovinin et al. (1994) using additional radial velocities measured with the CORAVELs and RVM. They derive elements very similar to the ones found here.

G175-39 $\left(04^{\mathrm{h}} 36^{\mathrm{m}} 06^{\mathrm{s}} \cdot 2,+55^{\circ} 24^{\prime} 45^{\prime \prime} ;\right.$ HIP 21433$): \epsilon=+2.4$ mas; an orbit was published by Tokovinin et al. (1994) with elements very similar to the ones found here.

G83-34 $\left(04^{\mathrm{h}} 48^{\mathrm{m}} 42^{\mathrm{s}}\right.$. $1,+18^{\circ} 42^{\prime} 33^{\prime \prime}$; HIP 22349): An orbit was published by Abt \& Willmarth (1987) with elements similar to the ones found here.

G191-23 $\left(05^{\mathrm{h}} 08^{\mathrm{m}} 26^{\mathrm{s}} .7,+55^{\circ} 25^{\prime} 12^{\prime \prime}\right.$; HIP 23922): Photocentric orbit, $P=672$ days, $a=8.5$ mas, and $i=69 \pm 16^{\circ}$; this solution needs to be reworked, given the spectroscopic orbit reported here.

G248-49 $\left(05^{\mathrm{h}} 46^{\mathrm{m}} 29 \mathrm{~s} \cdot 7,+74^{\circ} 36^{\prime} 36^{\prime \prime} ;\right.$ HIP 27246): $g_{\alpha}=$

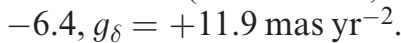

G108-53 $\left(07^{\mathrm{h}} 05^{\mathrm{m}} 04^{\mathrm{s}} .1,+01^{\circ} 23^{\prime} 50^{\prime \prime}\right.$; HIP 34164$): \epsilon=4.3$ mas.

G87-47 $\left(07^{\mathrm{h}} 35^{\mathrm{m}} 34^{\mathrm{s}} \cdot 0,+35^{\circ} 57^{\prime} 11^{\prime \prime}\right.$; HIP 36936$): \epsilon=10.4$ mas.

G112-54 $\left(07^{\mathrm{h}} 54^{\mathrm{m}} 34^{\mathrm{s}} 1,-01^{\circ} 24^{\prime} 44^{\prime \prime}\right.$; HIP 38625$)$ : visual companion, P.A. $=99^{\circ}$, sep $=4 " .88, \Delta H_{p}=4.04 \mathrm{mag}$; an orbit was published by Duquennoy \& Mayor (1991) with elements very similar to the ones found here.

G40-8 $\left(08^{\mathrm{h}} 08^{\mathrm{m}} 54^{\mathrm{s}} .3,+24^{\circ} 37^{\prime} 26^{\prime \prime} ;\right.$ HIP 39893$): \epsilon=4.8$ mas.

G41-34 $\left(09^{\mathrm{h}} 22^{\mathrm{m}} 46^{\mathrm{s}} .9,+11^{\circ} 16^{\prime} 18^{\prime \prime} ;\right.$ HIP 45990$): \epsilon=18.3$ mas.

G48-39 $\left(09^{\mathrm{h}} 49^{\mathrm{m}} 48^{\mathrm{s}} \cdot 4,+11^{\circ} 06^{\prime} 22^{\prime \prime}\right.$; HIP 48215$)$ : an orbit was published by Carquillat et al. (1983) with elements similar to the ones found here.

G58-23 $\left(10^{\mathrm{h}} 49^{\mathrm{m}} 52^{\mathrm{s}} \cdot 6,+20^{\circ} 29^{\prime} 29^{\prime \prime} ;\right.$ HIP 52958): $g_{\alpha}=$ $+17.8, g_{\delta}=-8.1$ mas yr$^{-2}$.

G44-45 $\left(10^{\mathrm{h}} 53^{\mathrm{m}} 23^{\mathrm{s}} .7,+09^{\circ} 44^{\prime} 21^{\prime \prime}\right.$; HIP 53238): $g_{\alpha}=$ $-11.6, g_{\delta}=+14.4$ mas yr $^{-2}$.

G56-15 $\left(11^{\mathrm{h}} 06^{\mathrm{m}} 31^{\mathrm{s}} .6,+14^{\circ} 15^{\prime} 44^{\prime \prime}\right.$; HIP 54299): visual companion, P.A. $=265^{\circ}$, sep $=2 ! \prime 084, \Delta H_{p}=1.57 \mathrm{mag}$.

G59-5 $\left(12^{\mathrm{h}} 13^{\mathrm{m}} 27^{\mathrm{s}} .7,+23^{\circ} 15^{\prime} 56^{\prime \prime}\right.$; HIP 59609): $g_{\alpha}=$

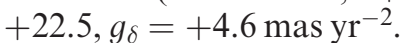

G121-75 (12 $19^{\mathrm{h}} 00^{\mathrm{s}} .7,+28^{\circ} 02^{\prime} 52^{\prime \prime}$; HIP 60061): $g_{\alpha}=$ $-8.9, g_{\delta}=-12.5$ mas yr$^{-2}$.

S138815 $\left(12^{\mathrm{h}} 29^{\mathrm{m}} 42^{\mathrm{s}} .7,-03^{\circ} 19^{\prime} 59^{\prime \prime} ;\right.$ HIP 60956): an orbit was published by Duquennoy \& Mayor (1991) and Jasniewicz \& Mayor (1988) with elements similar to those found here. 
G130-32 00:00:04.0+34:11:18 953

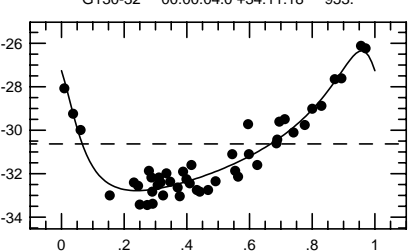

G1-21A $\quad 00: 45: 18.2+01: 40: 31 \quad 2037$

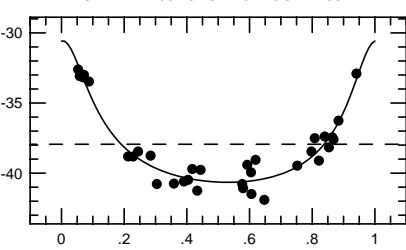

G172-38 $\quad 01: 05: 36.5+49: 27: 00 \quad 2554$

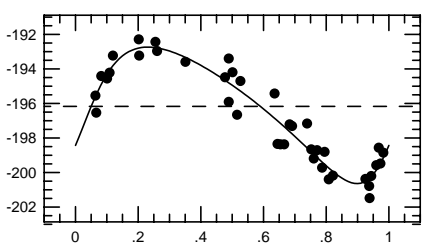

G172-61 01:34:22.6+48:44:27 1015 .

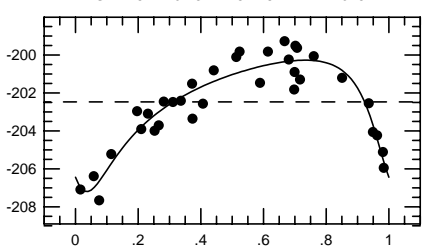

G72-58 $\quad 02: 08: 23.9+28: 18: 38 \quad 208.3$

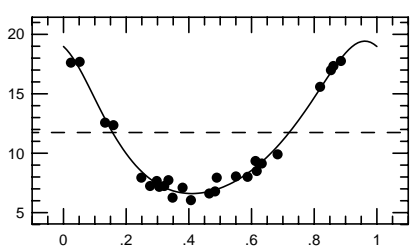

G173-59 02:33:53.7+49:30:21 2332.

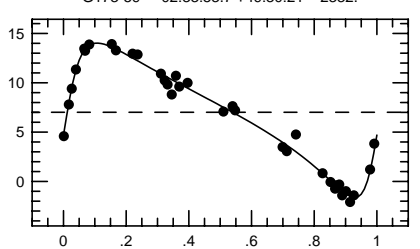

G36-32 02:44:22.1+27:40:08 $\quad 121.40$

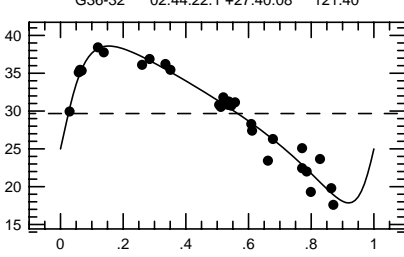

G77-56 03:29:18.6+01:58:31 179.02

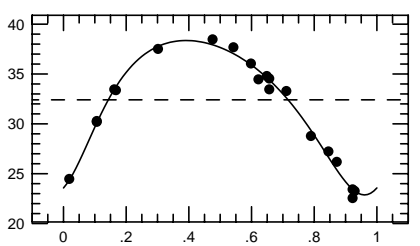

G265-1 00:08:50.8+86:47:16 $\quad 71.55$

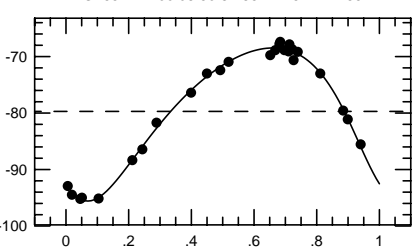

G32-49 $\quad 00: 47: 36.5+14: 38: 22 \quad 20.7097$

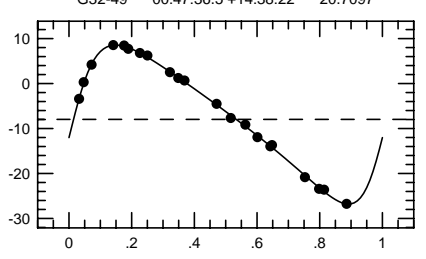

G34-16 01:16:36.4+18:31:21 274.56

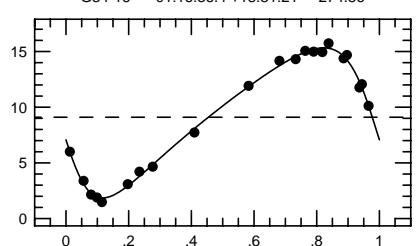

G72-18 01:37:56.1+32:21:00 1511.3

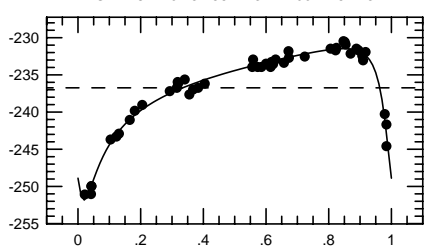

G72-59 02:08:23.9+28:18:18 87.754

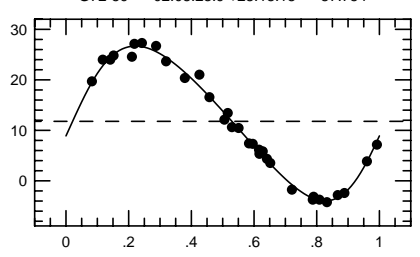

G220-13 02:35:32.8+59:47:32 905

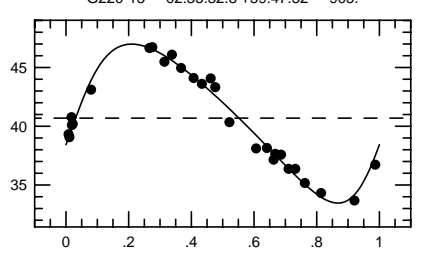

G4-45 $\quad 02: 54: 58.7+09: 54: 41 \quad 397.85$
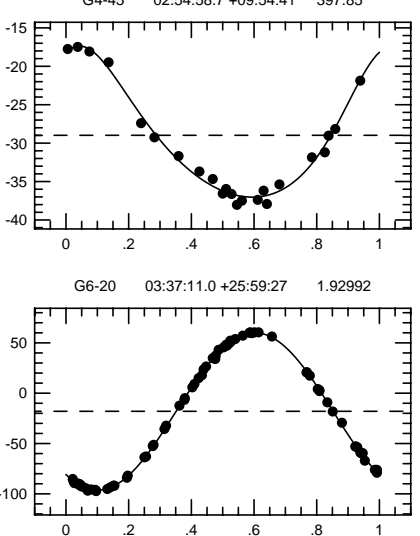

G171-58 $\quad 00: 25 \cdot 08.7+48 \cdot 02 \cdot 51 \quad 355.5$

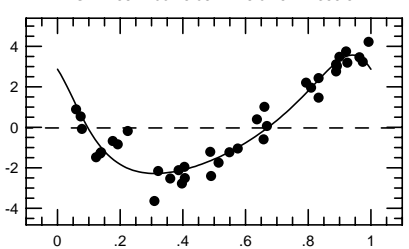

G32-56 00:53:14.1+19:08:48 4741.

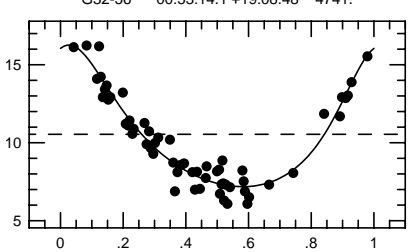

G172-49 $\quad 01: 21: 03.5+51: 59: 01 \quad 847.7$

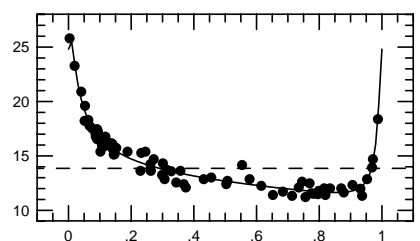

G71-27 01:41:56.2+01:54:09 4026

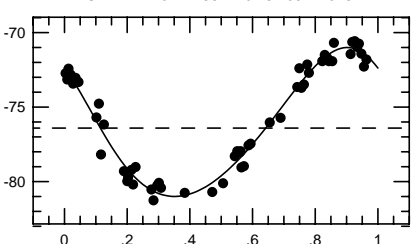

G4-16 02:22:34.0+15:31:07 1815.

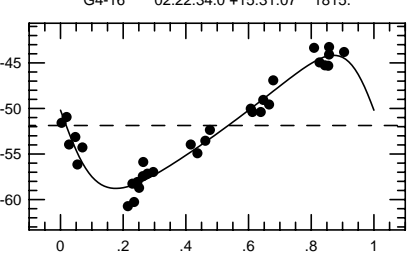

G134-40 02:37:41.4+43:59:34 227.9

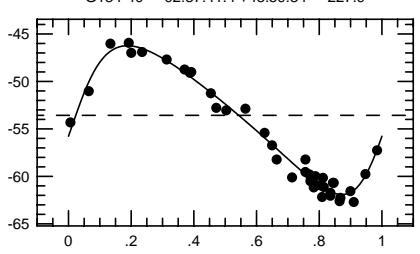

G5-27 03:18:27.1+15:10:38 3089.4

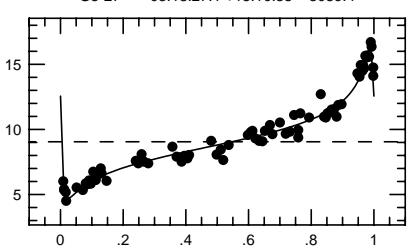

G95-57B $\quad 03: 47: 02.6+41: 25: 42 \quad 48.6521$

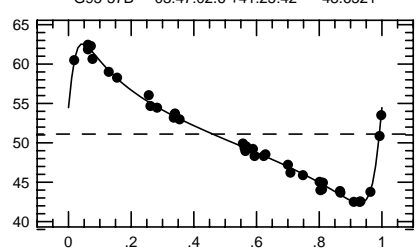

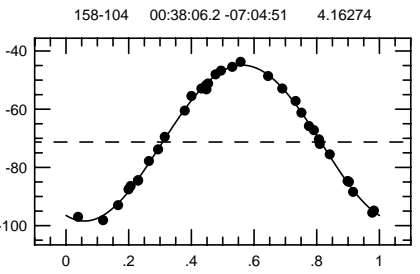
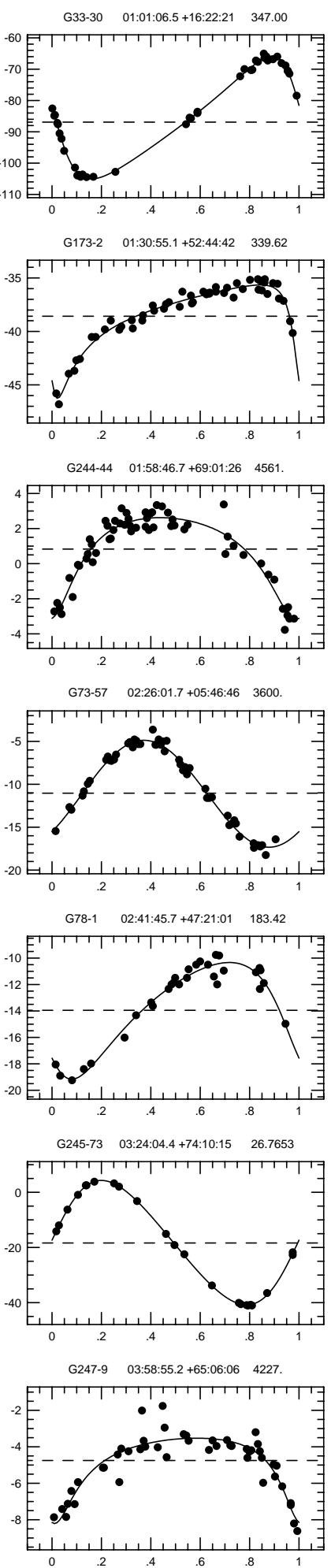

FIG. 4.-Solutions for 156 robust orbits 
G81-15 04:20:58.2+45:49:11 3900 .

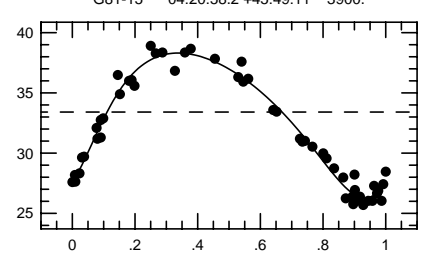

G248-27 04:56:36.3+72:57:05 7.5362

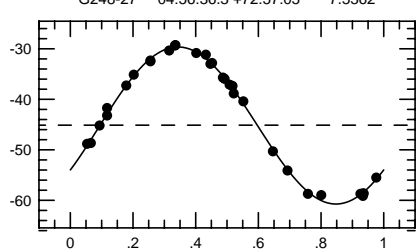

G248-49 05:46:29.7+74:36:36 4072.

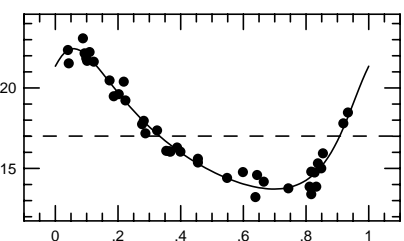

G106-46 06:25:08.0+00:38:56 4822.

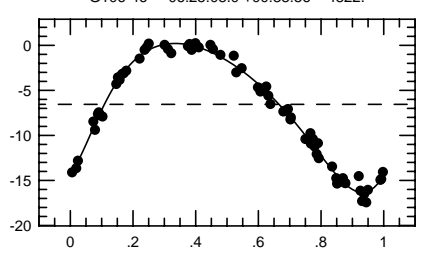

G108-58 07:10:02.2 -01:18:00 568.8

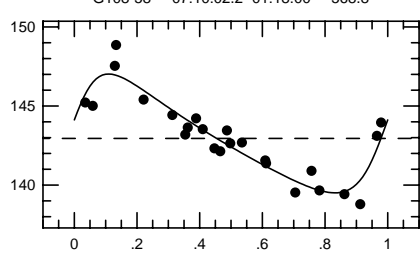

G89-14 $\quad 07 \cdot 22: 31.4+08: 49: 13 \quad 190.49$

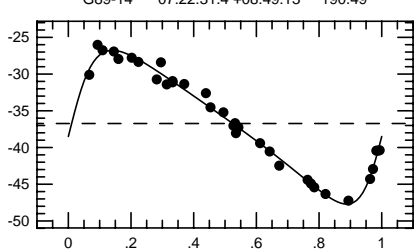

G40-5 $\quad 08: 04: 34.6+15: 21: 51 \quad 75.8905$
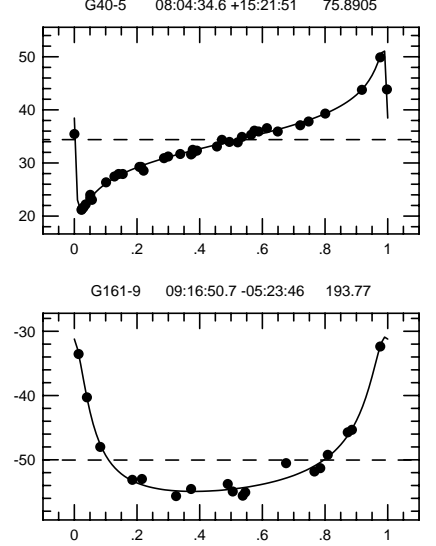

G175-39 $\quad 04: 36: 06.2+55: 24: 44 \quad 332.5$

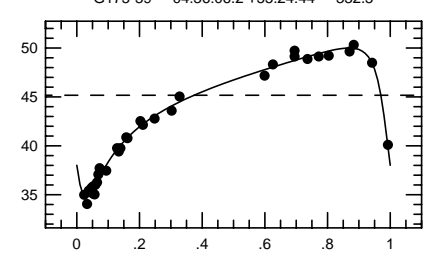

G191-23 05:08:26.6+55:25:12 1450

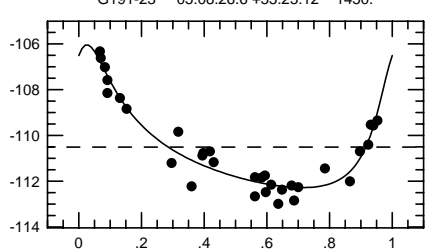

G99-52 06:02:04.5+00:17:56 558.3

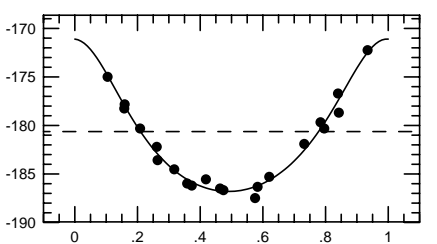

G87-20 07:03:04.8+38:08:32 85.182

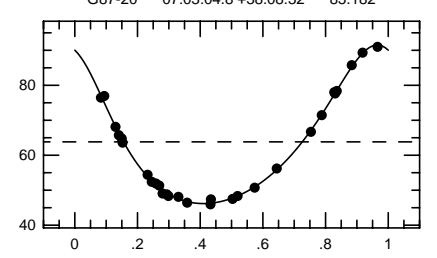

G88-11 $\quad 07: 10: 37.3+20: 26: 27 \quad 352.52$

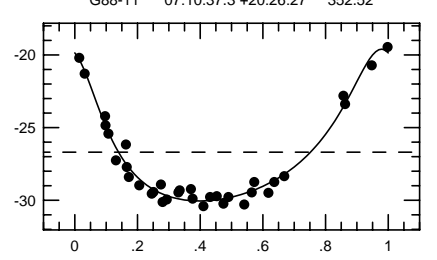

G87-47 $\quad 07: 35: 34.0+35: 57: 11 \quad 13.73134$

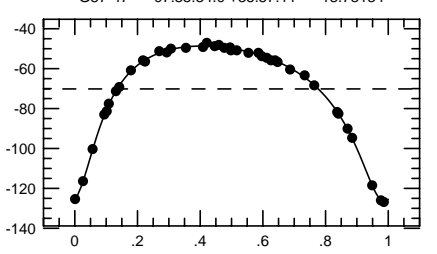

G40-8 $\quad 08: 08: 54.4+24: 37: 26 \quad 733.5$

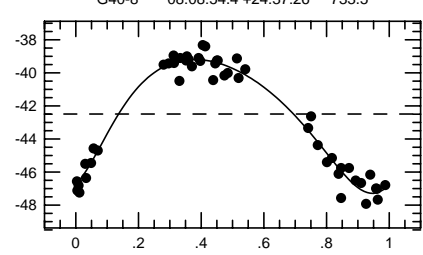

G46-31 09:17:04.0+03:01:29 1956

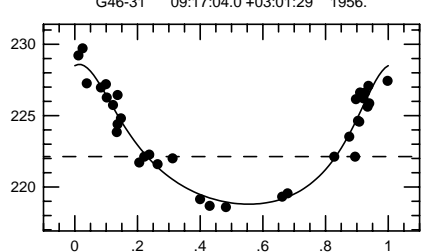

G8-46 04:37:49.9+20:04:05 637.6

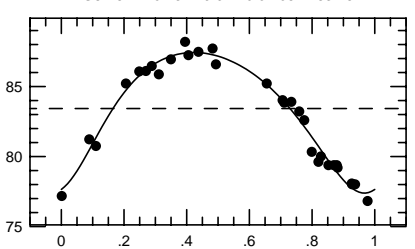

G84-39 05:12:45.2 +04:19:15 8.6630

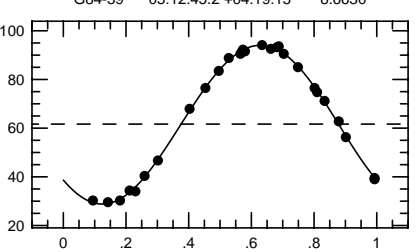

G249-37 06:06:24.6+63:50:06 48.936

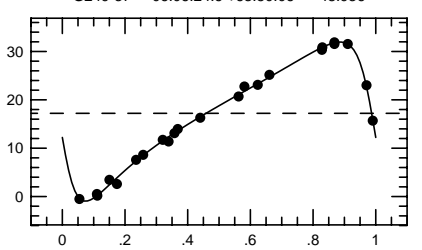

G108-53 07:05:04.1+01:23:50 612.3

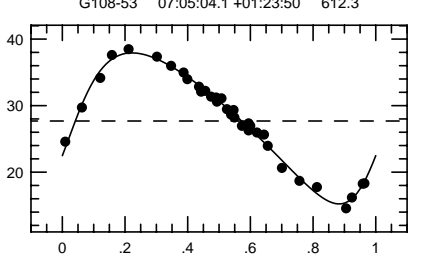

G87-35 07:20:44.4+29:20:38 1412.

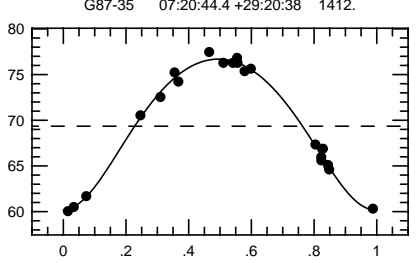

G112-54 07:54:34.1-01:24:44 450.4

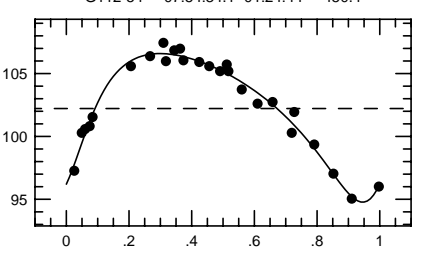

G40-14 $\quad 08: 16: 07.0+19: 41: 52 \quad 60.615$
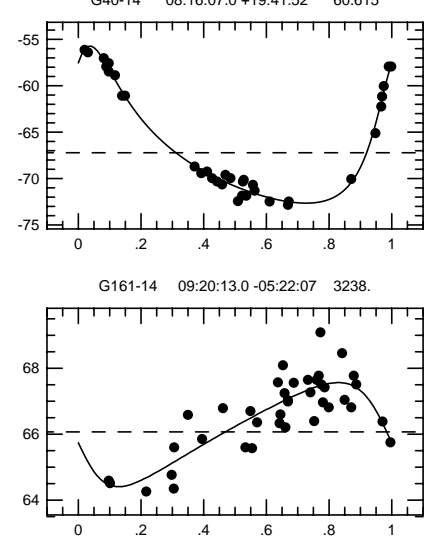

G83-34 04:48:42.1+18:42:33 45.4314

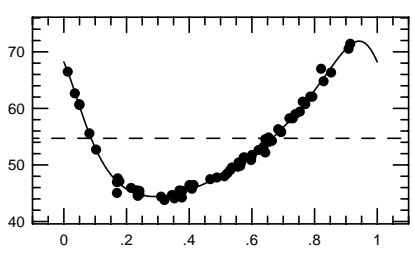

G99-30 05:43:44.1+03:34:58 229.41
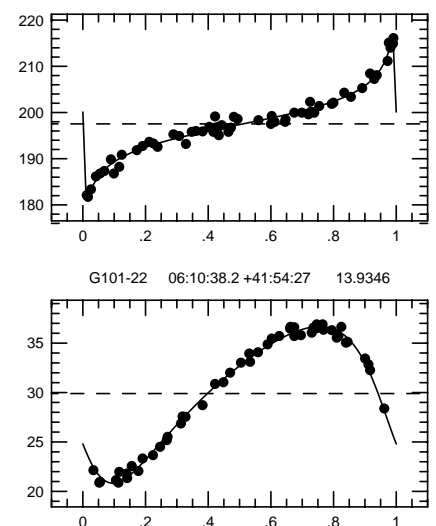

G88-5 07:06:07.5+18:38:07 $\quad 877.2$

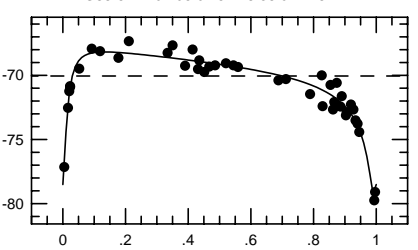

G250-43 07:20:47.6 +65:25:47 2431.

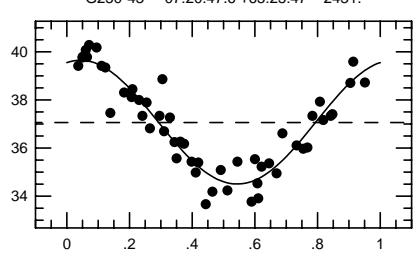

G40-2 07:59:33.9+20:50:38 3495

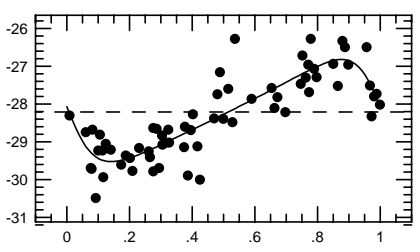

G9-42 $\quad 09: 00: 47.4+21: 27: 13 \quad 120.44$
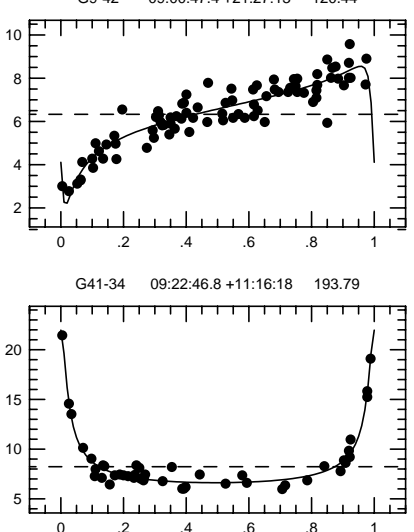

FIG. 4.-Continued 


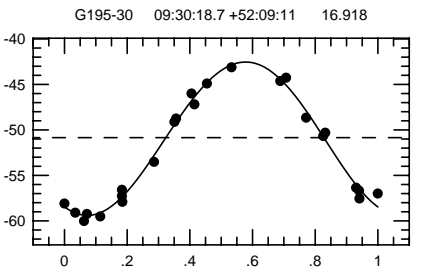

G53-30 10:12:29.8-00:38:13 1244

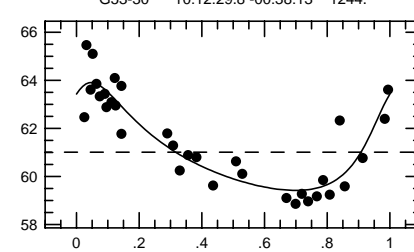

G44-45 10:53:23.8 +09:44:21 1841

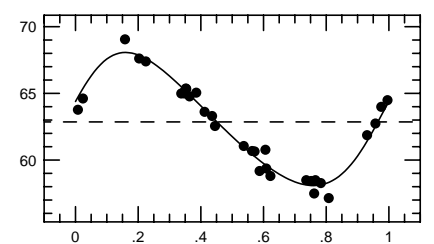

G147-36 11:17:14.5+29:34:14 6.57206

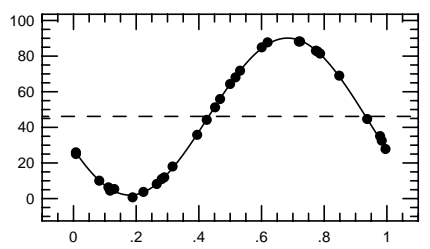

G59-5 12:13:27.7+23:15:56 2486

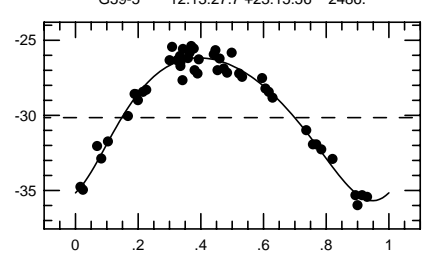

G59-27 12:36:39.4+27:28:28 60.012

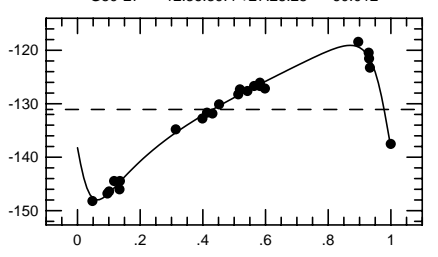

G255-32 13:21:47.6 +74:12:32 6376.

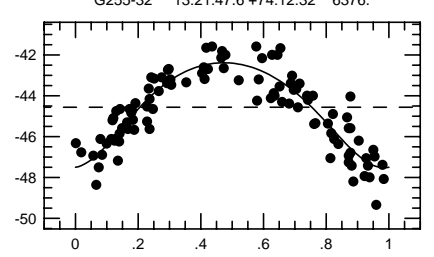

G62-44 13:31:39.9-02:19:02 1188.0

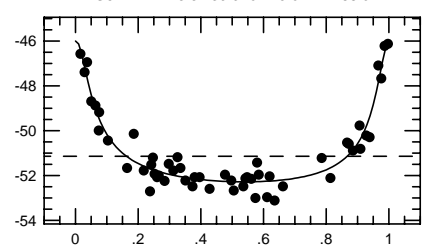

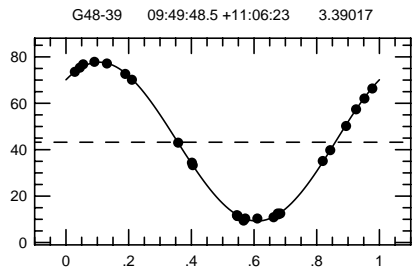

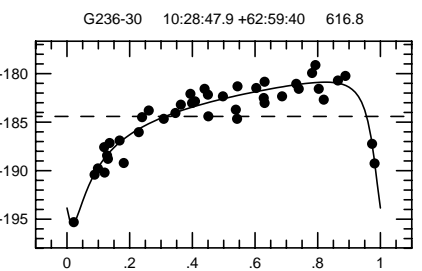

G161-84 09:51:39.4-03:49:57 2799.

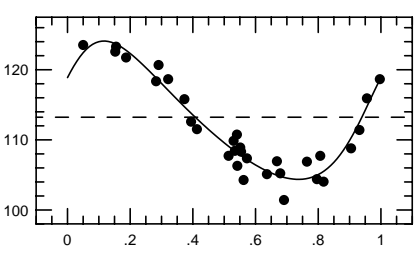

G58-23 10:49:52.6+20:29:30 1779.

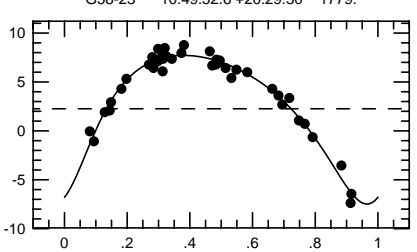

G58-39 11:03:26.8+18:08:15 2797.
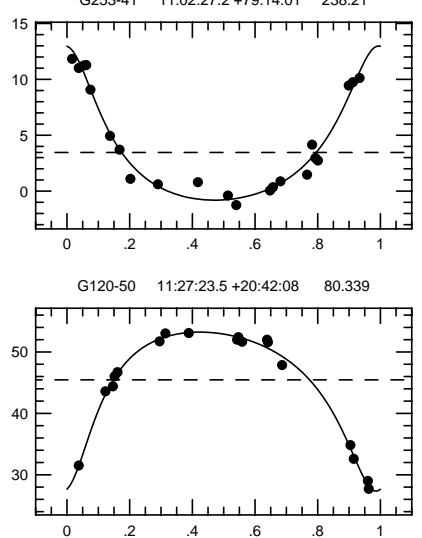

G123-14 12:13:30.1+47:10:07 2523.

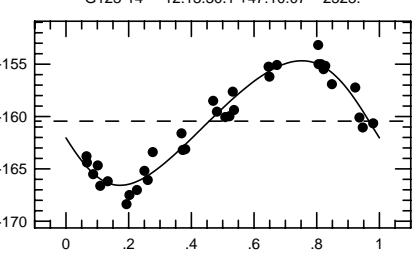

G60-47 12:55:15.9+07:49:57 1042.6

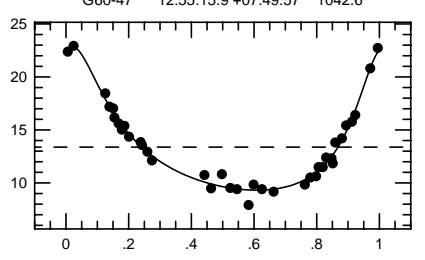

G63-27 13:24:36.8+14:51:25 3186.

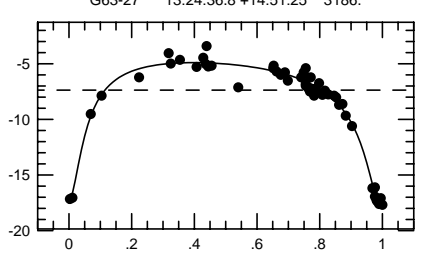

G62-52 13:36:01.7+01:12:07 46.420

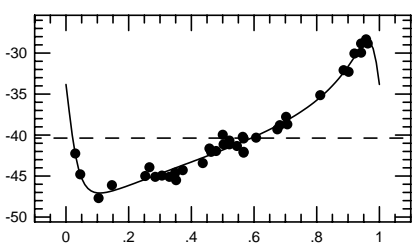

G147-58 11:30:22.3+35:50:30 $\quad 7.15122$

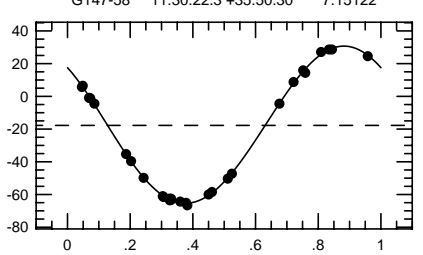

G121-75 12:19:00.8+28:02:52 2167.

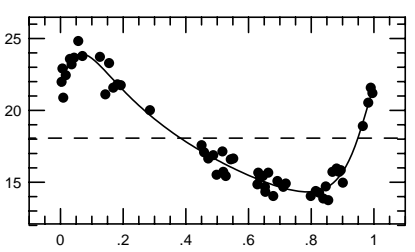

G62-30 13:19:55.3 +06:51:26 305.11

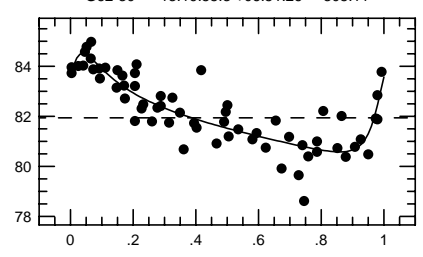

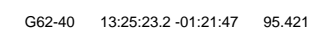

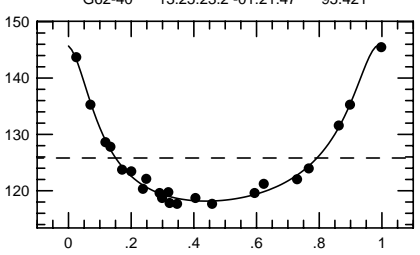

G165-22 13:38:01.9+39:10:41 11.58958

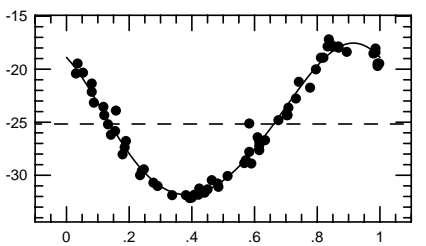

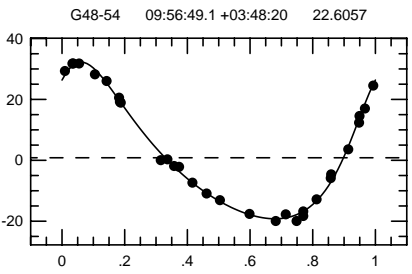
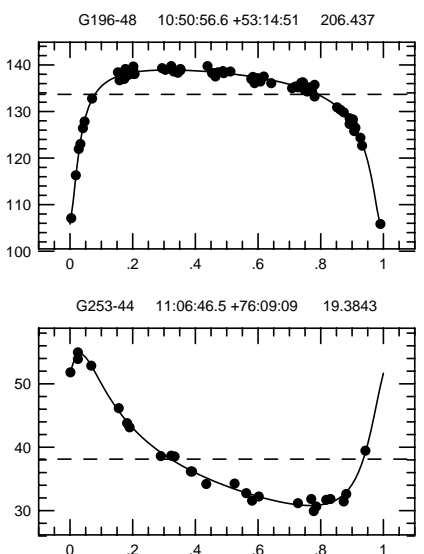

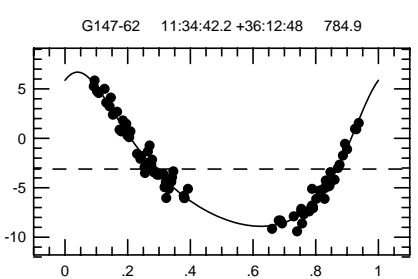

S138815 12:29:42.7-03:19:58 25.939
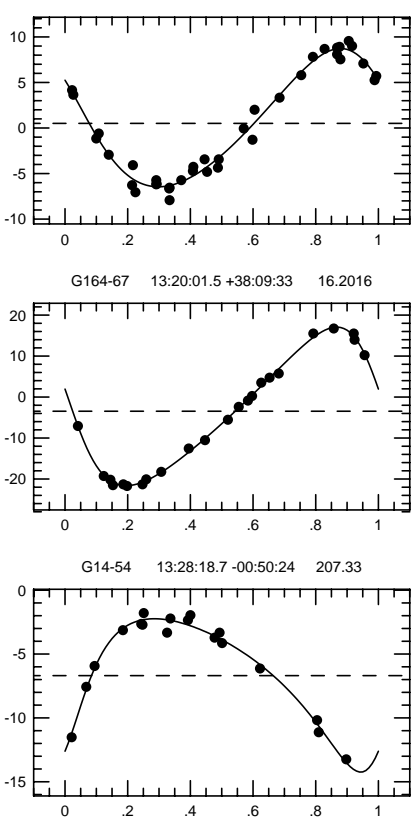

G62-61 13:40:27.0+02:09:06 14.49539

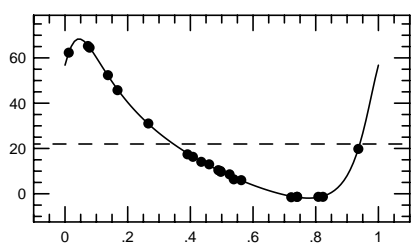

FIG. 4.-Continued 

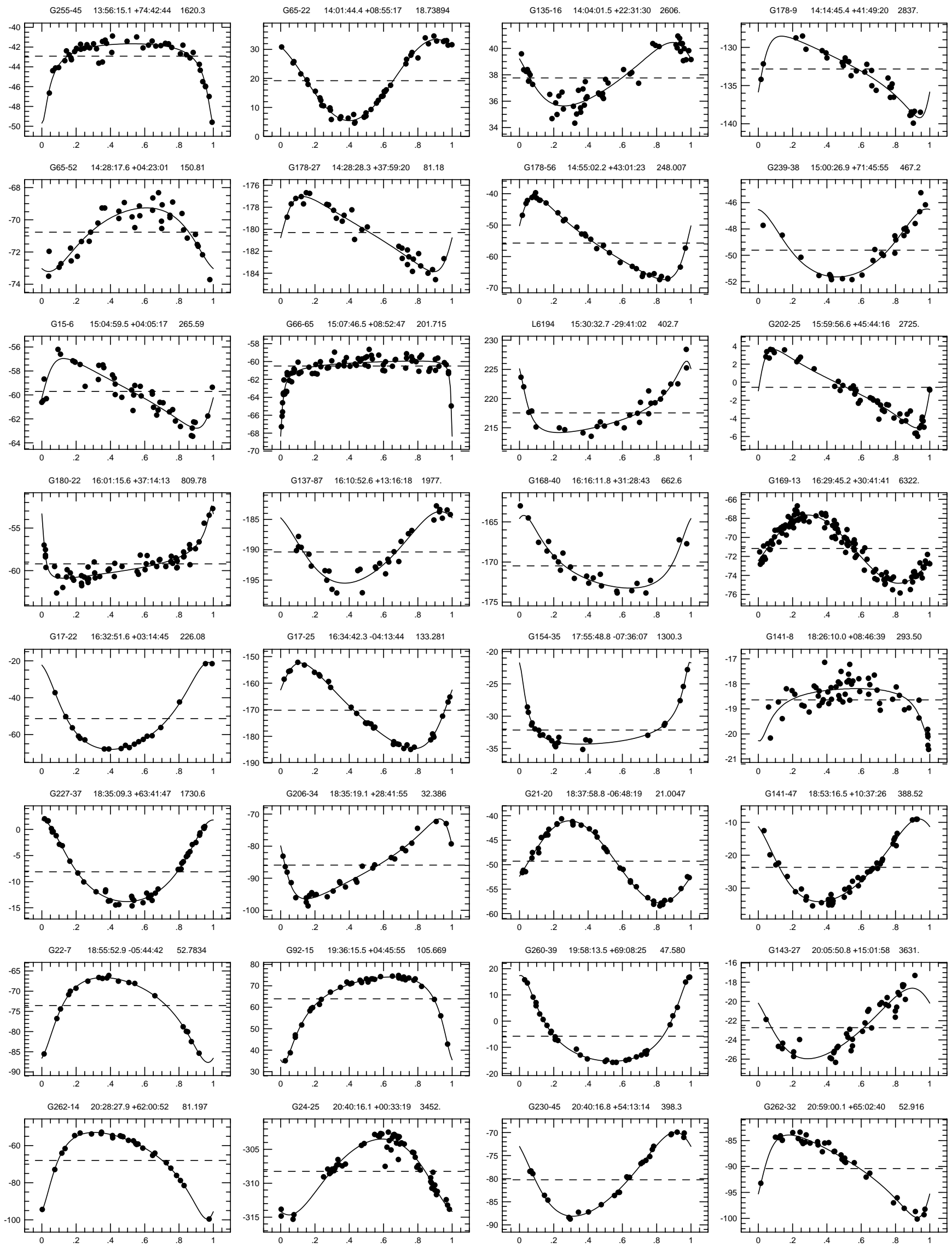

FIg. 4.-Continued 

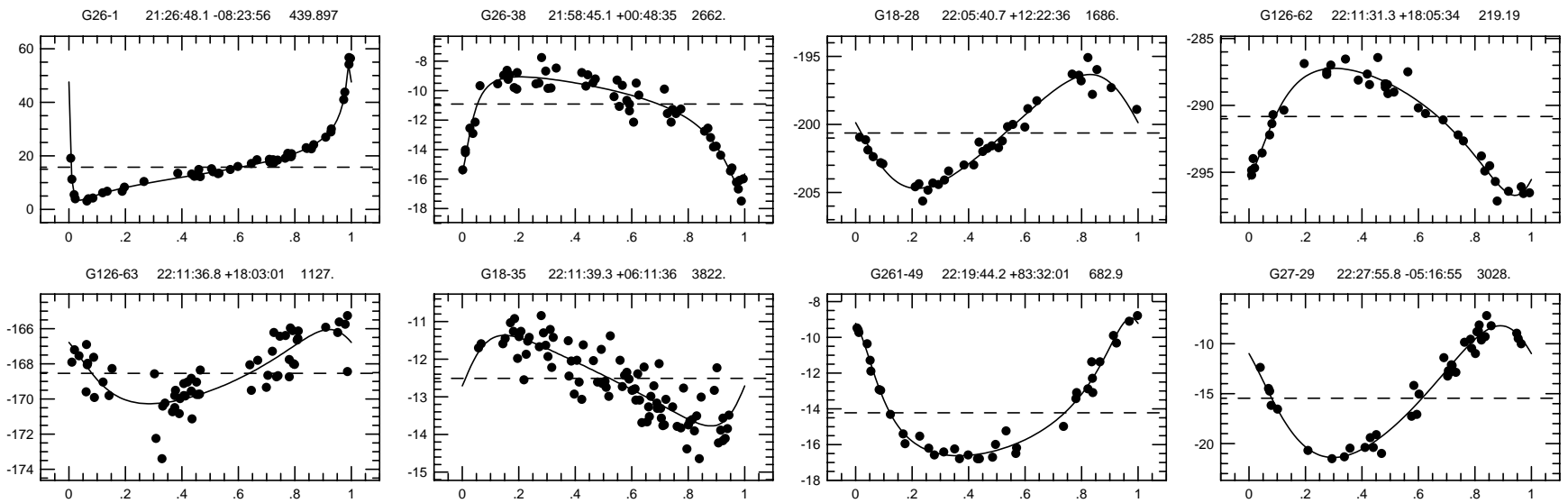

G127-33 22:28:02.0+24:56:16 3977
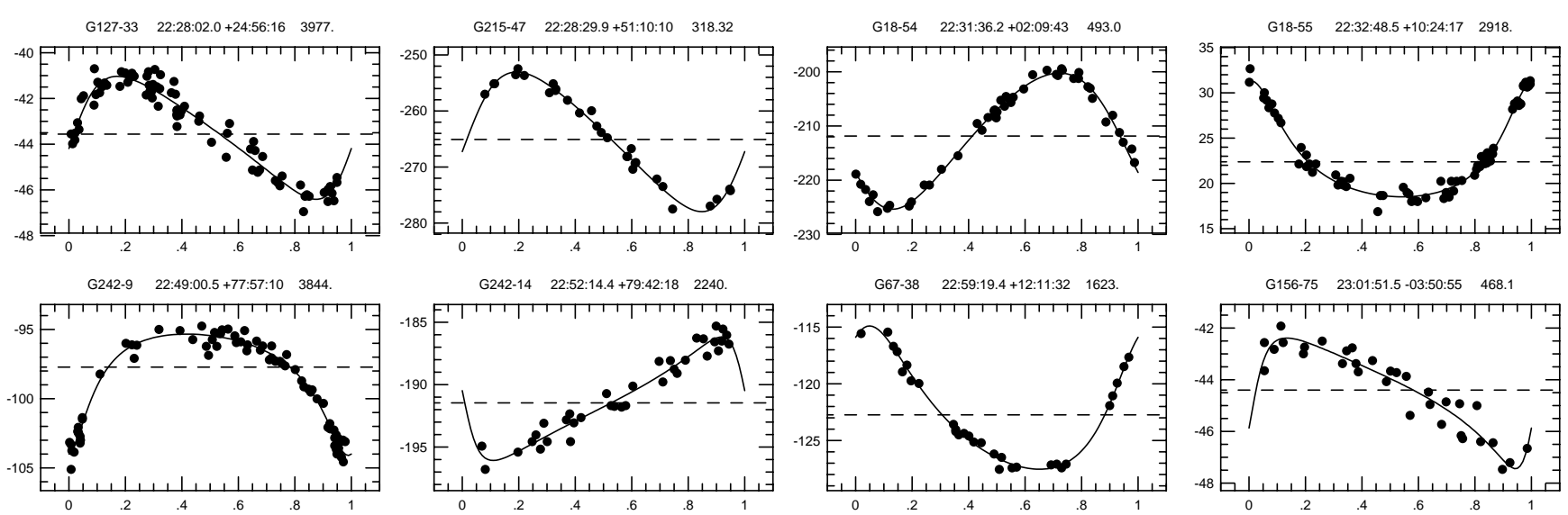

\begin{tabular}{ll} 
G190-10 $23: 07: 59.7+41: 51: 22 \quad 30.1712$ \\
\hline
\end{tabular}
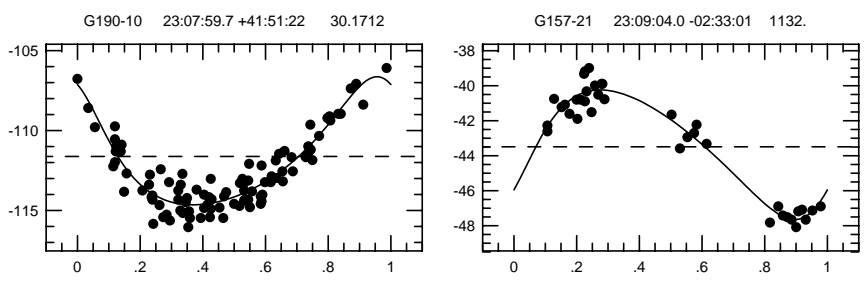

G190-15 23:13:38.8+39:25:02 184.05
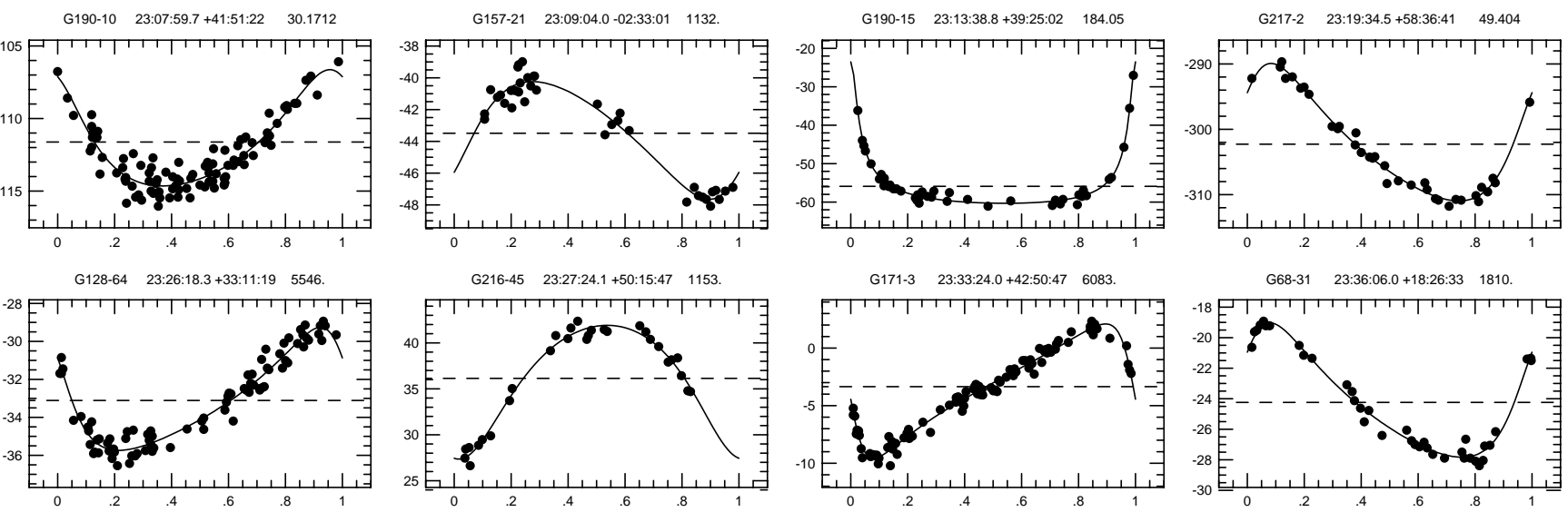

G171-3 23:33:24.0+42:50:47 6083.
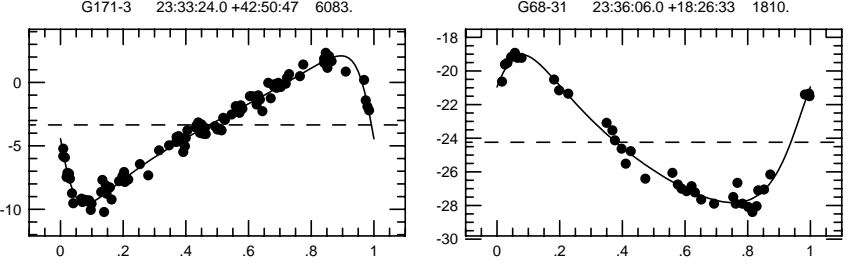

G130-10 23:46:09.3+35:14:38 $\quad 1756.0$
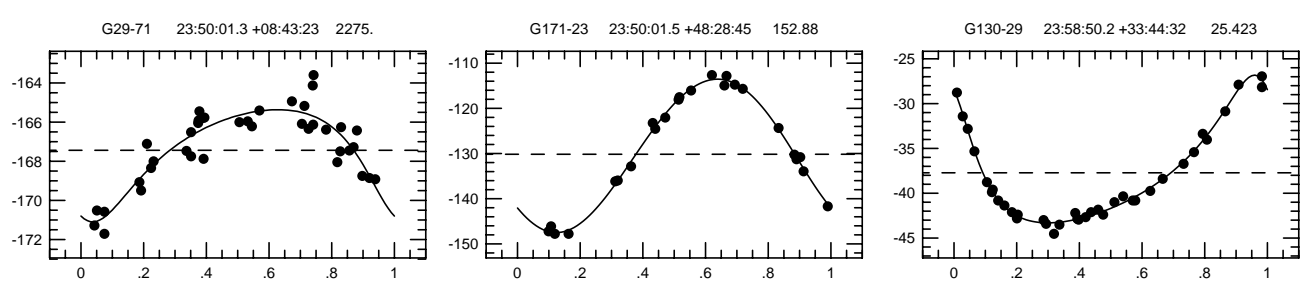

FIG. 4.-Continued

G164-67 (13 $20^{\mathrm{m}} 01^{\mathrm{s}} .5,+38^{\circ} 09^{\prime} 33^{\prime \prime}$; HIP 65049): an orbit was published by Griffin (1981) with elements very similar to the ones found here.

G62-44 $\left(13^{\mathrm{h}} 31^{\mathrm{m}} 39^{\mathrm{s}} \cdot 9,-02^{\circ} 19^{\prime} 03^{\prime \prime}\right.$; HIP 65982): visual companion, P.A. $=122^{\circ}$, sep $=0$ ". $167, \Delta H_{p}=1.8 \mathrm{mag}$.

G165-22 $\left(13^{\mathrm{h}} 38^{\mathrm{m}} 01^{\mathrm{s}} \mathrm{9}, \quad+39^{\circ} 10^{\prime} 41^{\prime \prime} ;\right.$ HIP 66514): visual companion, P.A. $=40^{\circ}$, sep $=1$ !" $770, \Delta H_{p}=2.21$ mag.
G255-45 ( $13^{\mathrm{h}} 56^{\mathrm{m}} 15^{\mathrm{s}} \cdot 1,+74^{\circ} 42^{\prime} 44^{\prime \prime}$; HIP 68072): $g_{\alpha}=$ $-7.3, g_{\delta}=-3.1$ mas yr $^{-2}$.

G65-22 $\left(14^{\mathrm{h}} 01^{\mathrm{m}} 44^{\mathrm{s}} 4,+08^{\circ} 55^{\prime} 17^{\prime \prime} ;\right.$ HIP 68527$): \epsilon=16.3$ mas.

G239-38 $\left(15^{\mathrm{h}} 00^{\mathrm{m}} 26^{\mathrm{s}} \cdot 9,+71^{\circ} 45^{\prime} 55^{\prime \prime} ;\right.$ HIP 73440$): \epsilon=2.3$ mas.

G17-22 $\left(16^{\mathrm{h}} 32^{\mathrm{m}} 51^{\mathrm{s}} .6,+03^{\circ} 14^{\prime} 45^{\prime \prime}\right.$; HIP 81023$)$ : photocentric orbit, $a=9.9 \pm 1.6$ mas, $i=109^{\circ} \pm 15^{\circ}$; an orbit 
was published by Johnson \& Mayor (1986) with elements very similar to the ones found here. They conclude that the companion is itself a short-period binary.

G17-25 $\left(16^{\mathrm{h}} 34^{\mathrm{m}} 42^{\mathrm{s}} .3,-04^{\circ} 13^{\prime} 44^{\prime \prime}\right.$; HIP 81170$)$ : an orbit was published by Duquennoy \& Mayor (1991) and Mayor \& Turon (1982) with elements very similar to the ones found here. The velocity of the secondary was detected by Mazeh et al. (1997), who derived a mass ratio of $0.57 \pm 0.02$.

G141-19 $\left(18^{\mathrm{h}} 33^{\mathrm{m}} 16^{\mathrm{s}} .8,+13^{\circ} 09^{\prime} 25^{\prime \prime}\right.$; HIP 90957): an orbit was published by Jasniewicz \& Mayor (1986) with elements very similar to the ones found here.

G227-37 $\left(18^{\mathrm{h}} 35^{\mathrm{m}} 09^{\mathrm{s}} .3,+63^{\circ} 41^{\prime} 47^{\prime \prime}\right.$; HIP 91115$)$ : visual companion, P.A. $=23^{\circ}$, sep $=19^{\prime \prime} 950, \Delta H_{p}=2.70$ mag.

G141-47 $\left(18^{\mathrm{h}} 53^{\mathrm{m}} 16^{\mathrm{s}} \cdot 1,+10^{\circ} 37^{\prime} 21^{\prime \prime}\right)$ : Erroneously listed as double-lined in Paper VI.

G156-75 $\left(23^{\mathrm{h}} 01^{\mathrm{m}} 51^{\mathrm{s}} .5,-03^{\circ} 50^{\prime} 55^{\prime \prime}\right.$; HIP 113718$)$ : photocentric orbit, $a=11.1 \pm 2.5$ mas, $i=58^{\circ} .9 \pm 5^{\circ} .8$; an orbit was published by Tokovinin et al. (1994) with elements very similar to the ones found here.

G157-21 $\left(23^{\mathrm{h}} 09^{\mathrm{m}} 04^{\mathrm{s}} 0,-02^{\circ} 33^{\prime} 01^{\prime \prime}\right.$; HIP 114313$): g_{\alpha}=$ $+8.0, g_{\delta}=+37.1$ mas yr $^{-2}$.

G171-23 $\left(23^{\mathrm{h}} 33^{\mathrm{m}} 24^{\mathrm{s}} 0,+42^{\circ} 50^{\prime} 48^{\prime \prime}\right.$, HIP 116259$)$ : visual companion, P.A. $=341^{\circ}$, sep $=0$ ". $195, \Delta H_{p}=2.61 \mathrm{mag}$.

G68-31 $\left(23^{\mathrm{h}} 36^{\mathrm{m}} 06^{\mathrm{s}} 0,+18^{\circ} 26^{\prime} 33^{\prime \prime}\right.$; HIP 116478$)$ : photometric variable, amplitude $=0.10$ mag; visual companion, P.A. $=348^{\circ}$, sep $=5 " .307, \Delta H_{p}=1.30$ mag.

G130-10 $\left(23^{\mathrm{h}} 46^{\mathrm{m}} 09^{\mathrm{s}} \cdot 4,+35^{\circ} 14^{\prime} 37^{\prime \prime}\right.$; HIP 117229): $g_{\alpha}=$

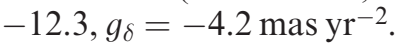

The details for 15 preliminary orbital solutions are presented in Table 5 and Figure 5. These are binaries that we continue to observe because the phase coverage is incomplete and/or the solution is not robust to the removal of a few observations.

In Figure 6, we plot the velocity histories for 17 more stars that are definitely spectroscopic binaries, but for which we do not yet have a reliable estimate of the period. All 17 systems have $P\left(\chi^{2}\right)$ values smaller than 0.0001 and in addition show clear trends in their velocity histories.

The IAU radial velocity standard star HD 114762 has a single-lined spectroscopic orbit with a minimum mass for the companion of about $0.01 M_{\odot}$ (cf. Latham et al. 1989; Mazeh, Latham, \& Stefanik 1996; Marcy et al. 1999). It is a member of our proper-motion sample, but we have not included it in this paper because its orbit has a substantially lower amplitude than we would expect to detect for binaries in the rest of the sample. The sensitivity for the detection of binaries among the IAU standards is better because we have observed them much more extensively. Furthermore, we believe that the argument offered by Marcy et al. (1999) that the orbit of HD 114762 is likely to be nearly face-on just because it was included in the full Carney-Latham sample of 1464 stars is fundamentally incorrect, and the unseen companion of HD 114762 should not be ruled out as a giant planet on this basis.

In addition to HD 114762, there are at least 10 more binaries in our sample for which the minimum mass of the companion is below the nominal substellar limit of 0.075

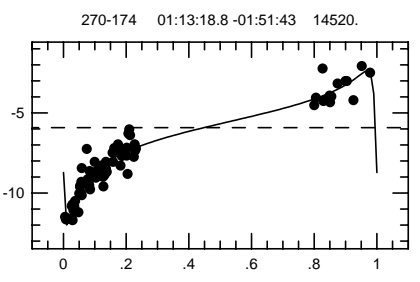

G115-52 09:06:50.5 +47:06:51 5614

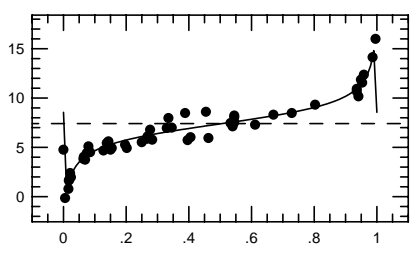

G196-42 10:46:10.6+56:28:16 8573

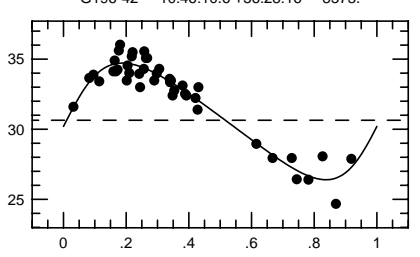

G201-44 15:13:57.3+53:51:51 7061.

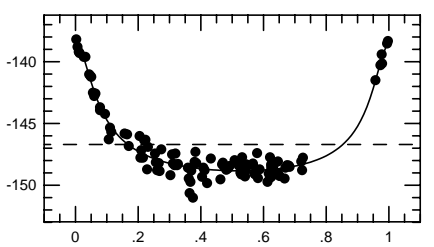

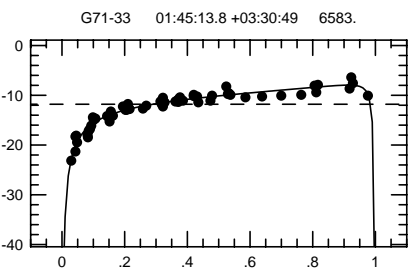

G116-9 09:09:30.6+38:53:34 1732

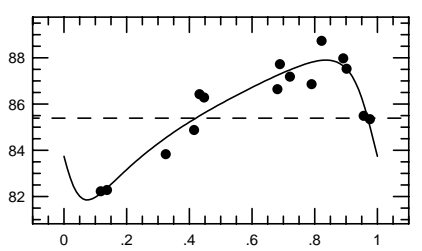

G56-15 11:06:31.5+14:15:44 6089.

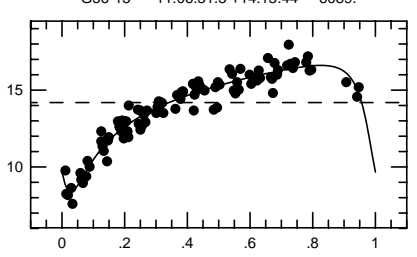

G202-35 16:14:57.4+49:46:06 14102.

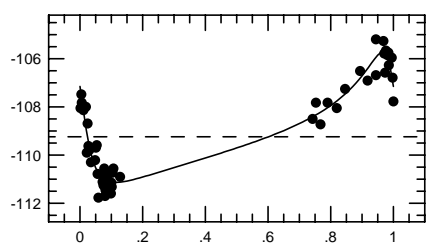

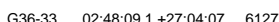

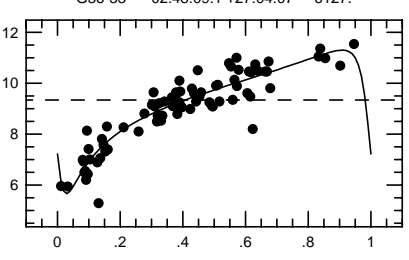

G49-19 09:38:50.6+28:24:08 5067

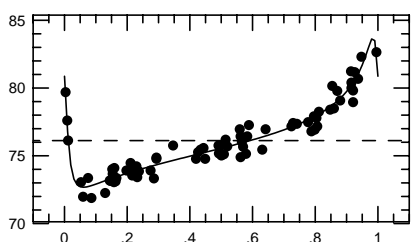

G197-56 12:21:28.1+61:44:50 6864

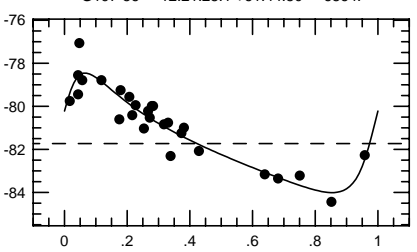

G217-8 23:26:32.8+60:37:42 9632.

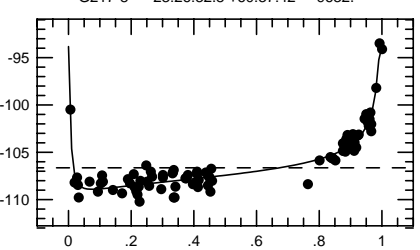

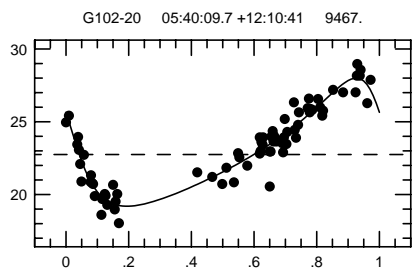

G42-30 09:59:10.1+14:47:00 $\quad 797.3$

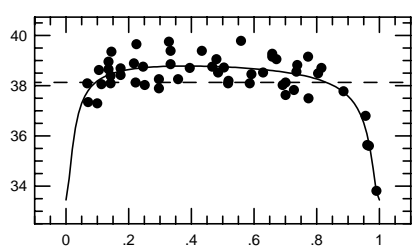

G61-30 13:06:35.7+11:02:40 2310.3

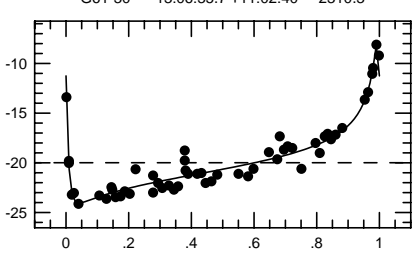

FIG. 5.- Solutions for 15 preliminary orbits 
TABLE 6

Substellar Secondary Minimum Masses

\begin{tabular}{|c|c|c|c|c|}
\hline Star & R.A. (J2000.0) & Decl. (J2000.0) & $\begin{array}{c}\mathscr{M}_{\mathrm{A}} \\
\left(M_{\odot}\right)\end{array}$ & $\begin{array}{c}\mathscr{M}_{\mathrm{B} \min } \\
\left(M_{\odot}\right)\end{array}$ \\
\hline G9-42.......... & 090047.4 & +212713 & 0.77 & 0.038 \\
\hline G62-30....... & 131955.3 & +065126 & 0.68 & 0.040 \\
\hline G165-22 ...... & 133801.9 & +391041 & 0.82 & 0.070 \\
\hline G65-52 ....... & 142817.6 & +042301 & 0.62 & 0.036 \\
\hline G178-27 ...... & 142828.3 & +375920 & 0.68 & 0.051 \\
\hline G15-6......... & 150459.5 & +040517 & 0.67 & 0.064 \\
\hline G66-65 ........ & 150746.5 & +085247 & 0.70 & 0.043 \\
\hline G141-8....... & 182610.0 & +084639 & 0.77 & 0.023 \\
\hline G18-35....... & 221139.3 & +061136 & 0.75 & 0.072 \\
\hline G190-10..... & 230759.7 & +415122 & 0.60 & 0.044 \\
\hline
\end{tabular}

$M_{\odot}$. These systems are summarized in Table 6, where we list the primary mass estimate, $\mathscr{M}_{\mathrm{A}}$, from Paper XII (when available) and the corresponding minimum mass for the companion, $\mathscr{M}_{\mathrm{B} \min }$, for $\sin i=1$. The implications for the distribution of secondary masses near the substellar limit will be discussed in a future paper.

\section{HALO VERSUS DISK BINARY POPULATIONS}

In this section, we illustrate that there are no obvious differences in the characteristics of the binaries in the halo versus the disk populations. To show that our binaries have more or less the same distribution with kinematics and metallicity as our constant stars, we plot in Figure 7 the Galactic $V$ velocity (more correctly, the planar Galactic specific angular momentum directed toward a Galactic longitude of $90^{\circ}$ and a Galactic latitude of $0^{\circ}$ ) versus the metallicity, $[\mathrm{m} / \mathrm{H}]$, derived from our spectra (Carney et al. 1987), quoted as the log of a representative metal abundance compared to solar. The top panel shows 1013 stars with no detected velocity variation, while the bottom panel shows 205 stars identified as spectroscopic binaries. The total number of stars in the two panels is less than 1359 because we do not have metallicities and/or $V$ velocities for some of our stars. For this comparison, we have included all the stars identified as binaries, whether or not orbital solutions were available, and for which $V$ and $[\mathrm{m} / \mathrm{H}]$ values were available.

Our procedures for deriving the Galactic $V$ velocity have been updated since the publication of Paper XII. In brief, when Hipparcos parallaxes became available, they were used in preference to the photometric distances presented in Paper XII. Furthermore, the photometric distance scale has been adjusted to match the Hipparcos distance scale, and revised photometric distances have been derived for the stars without Hipparcos distances. In addition, we have worked to identify subgiants, so that their photometric distances could be determined more accurately. This is work in progress that will be reported in a future paper.

The halo population (e.g., Carney et al. 1990, 1996; Carney, Latham, \& Laird 1989, 1990; Laird et al. 1988) is illustrated in Figure 7 by the broad distribution of objects centered roughly at $[\mathrm{m} / \mathrm{H}]=-1.5$ and $V=-220 \mathrm{~km}$ $\mathrm{s}^{-1}$, while the disk populations are concentrated close to $[\mathrm{m} / \mathrm{H}]=0$ and $V=0 \mathrm{~km} \mathrm{~s}^{-1}$. Figure 7 conveys the impression that the single-lined binaries and the constant stars have very similar distributions, and that binaries have more or less the same frequency in the halo as in the disk populations.

To illustrate this point in a different way, we separate the halo from the disk populations using the dotted contour plotted on Figure 7. This contour was drawn by eye and is somewhat arbitrary. In Figure 8, we plot the cumulative distributions for the binary fraction in the halo and disk populations as a function of the log of the period, where we have included only the 171 single-lined binaries reported in this paper and the 34 double-lined binaries reported in Paper $\mathrm{XV}$ and have not included systems recognized as triples. For the binary fraction, we use the number of binary systems relative to the total number of systems, i.e., constant stars plus binaries. The two distributions are quite similar, and the $\mathrm{K}-\mathrm{S}$ test finds that the probability of getting this much difference or more by accident for two parent distributions that are identical is about 0.7 when the full range of periods is considered. The overall frequency of halo spectroscopic binaries comes out marginally lower than the disk binaries, $14.5 \% \pm 1.8 \%$ versus $15.6 \% \pm 1.5 \%$, which is within the uncertainties. This might be the result of an observational bias, because halo binaries have lower metallicity and therefore weaker lines, with a corresponding poorer velocity precision and higher threshold for the detection of binaries.

There is another reason why this binary frequency for the halo may be slightly underestimated. We have not included the results for 10 metal-poor field blue stragglers in this paper, despite the fact that they belong to the full sample, because those results have already been published (Paper XIV). Seven of the blue stragglers meet our criteria for the halo, and four of these have orbital solutions. Two of the three disk blue stragglers are spectroscopic binaries. When the blue stragglers are included in the analysis, the revised binary frequencies are even closer to each other, $15.1 \pm 1.8$ for the halo compared with $15.8 \pm 1.5$ for the disk populations. Moreover, the distributions shown in Figure 8 suggest that the lower overall frequency of halo binaries comes largely from the region with periods shorter than a few hundred days. This is consistent with our speculation in Paper XIV that field metal-poor blue stragglers are the product of stable mass transfer, resulting in the evolution of shortperiod binaries into longer period binaries. When only the binaries with periods shorter than a few hundred days are considered, the K-S test finds that the probability of getting this much difference or more by accident is in the range $0.1-0.2$.

We do not have metallicities and/or Galactic $V$ velocities for about $10 \%$ of our binaries and therefore cannot assign them to the halo or disk populations. Thus, the binary frequencies quoted above are based on an incomplete sample. Of course, there is an additional, very large incompleteness for the long-period binaries that we have missed. Nevertheless, our comparison of the binaries in the halo and the disk may actually be valid for periods up to 10 or 20 years, because our observing protocols did not include any biases that would favor one population over the other, except that our ability to detect lowamplitude spectroscopic binaries is poorer for more metal-poor stars. This bias is most likely to show up for binaries with orbital amplitudes near our detection threshold, for example, at the longest periods.

From the point of view of binary formation, the main distinction between the halo and the disk populations is that the halo is much more metal-poor. The fact that the period 
G171-57 00:25:04.2+45:41:19
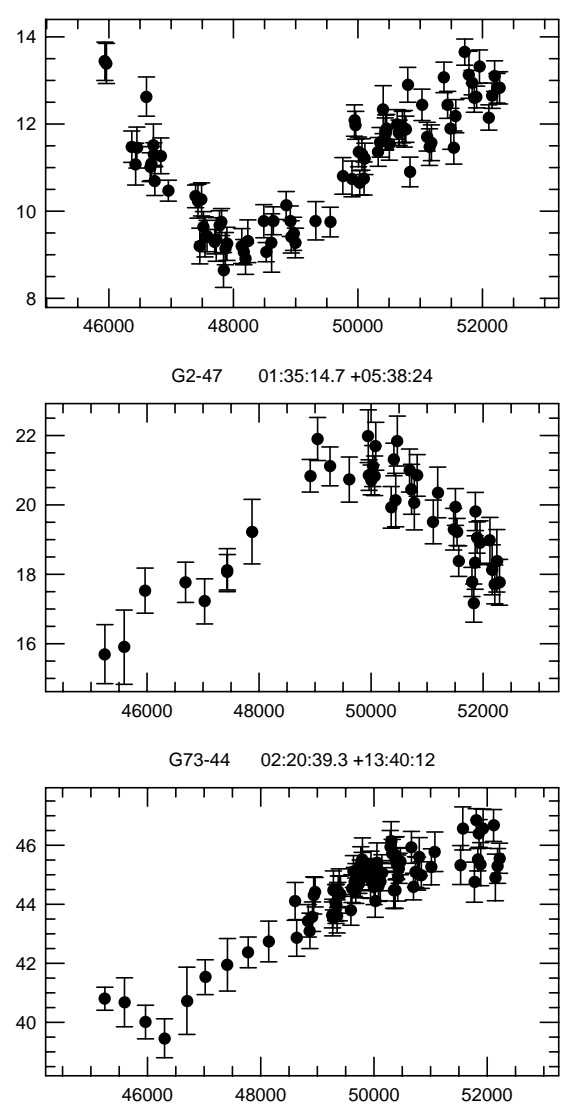

G82-12 04:20:14.5 -03:45:05

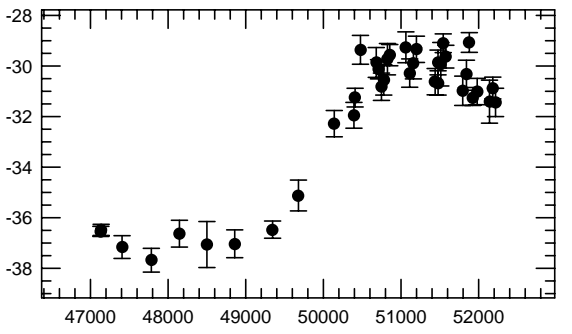

G250-23 06:50:19.5 +60:55:44
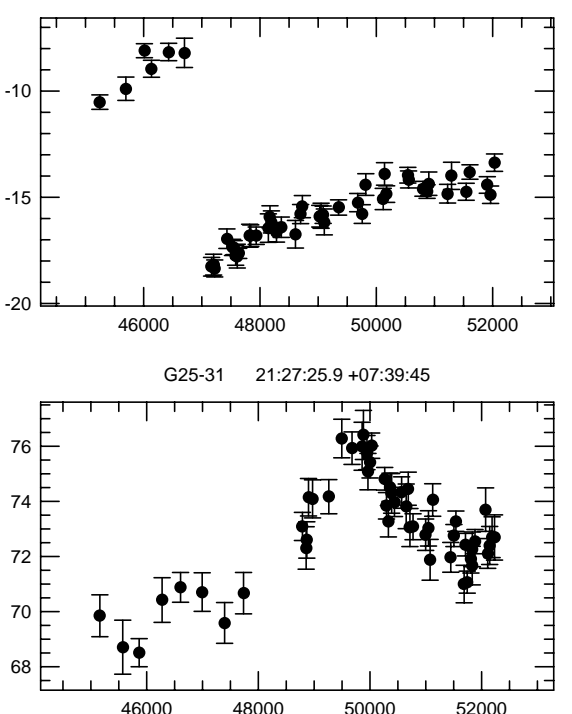

G1-9 00:39:13.2 +03:08:02

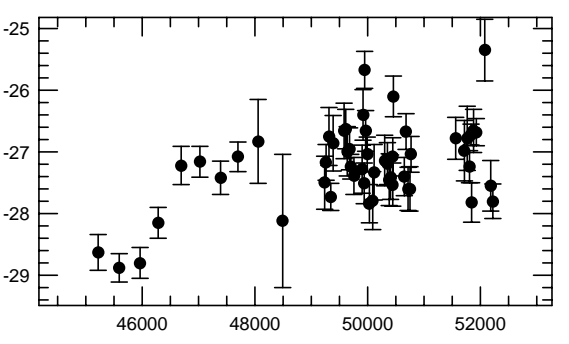

G3-10 01:38:14.1 +17:49:45

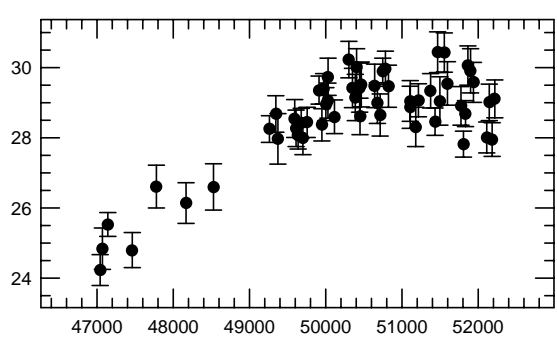

G37-34 03:19:39.8+33:35:55

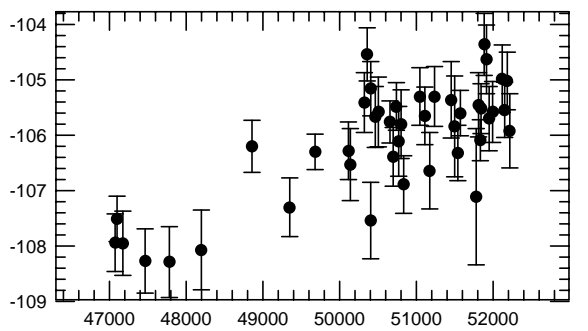

G100-60 06:08:27.9 +22:26:11

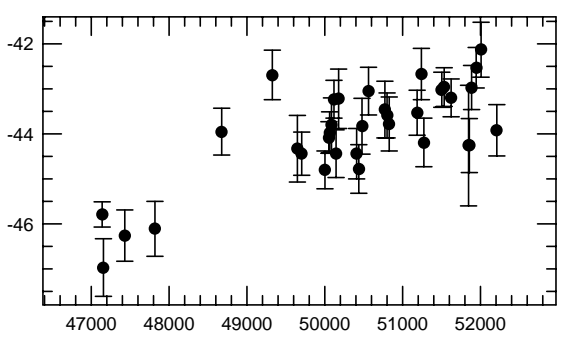

G65-43 14:18:12.2 +12:44:29
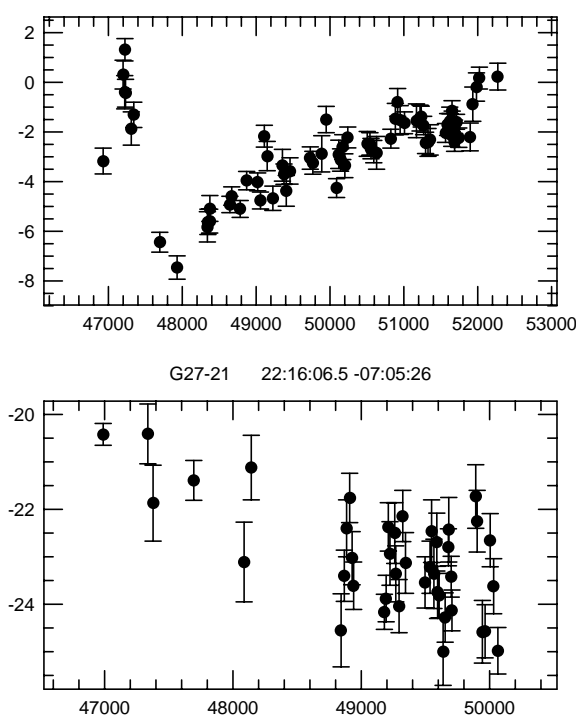

G172-46 01:16:38.2 +51:51:21
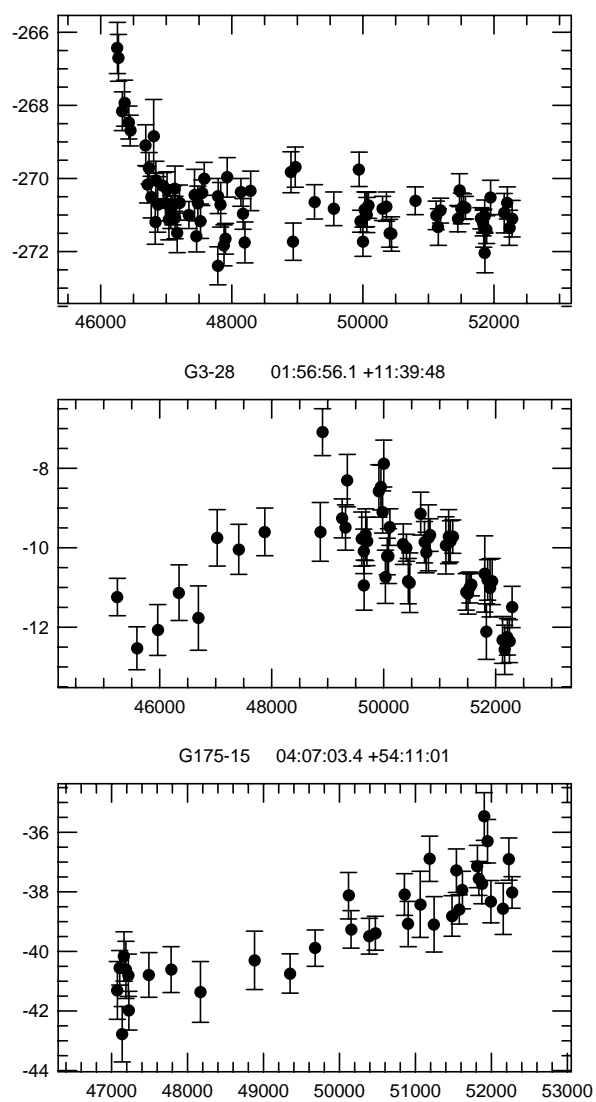

G103-52 06:42:47.2 +30:58:12
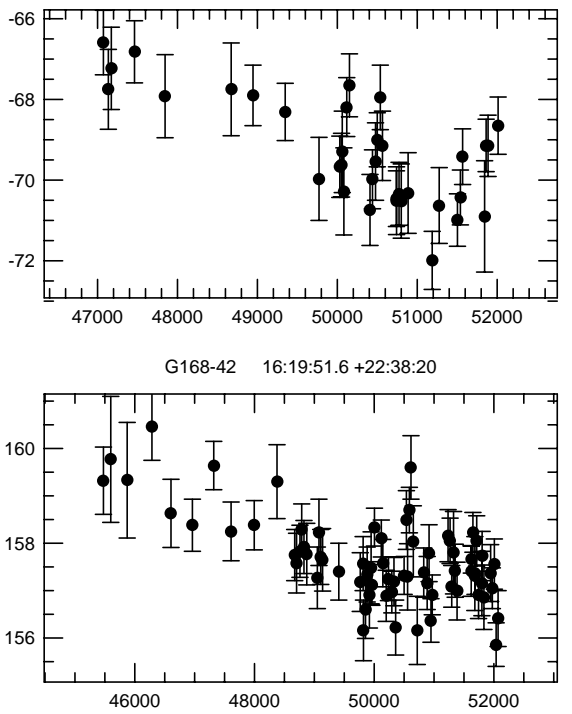

FIG. 6.-Velocity histories for 17 spectroscopic binaries with unknown periods 

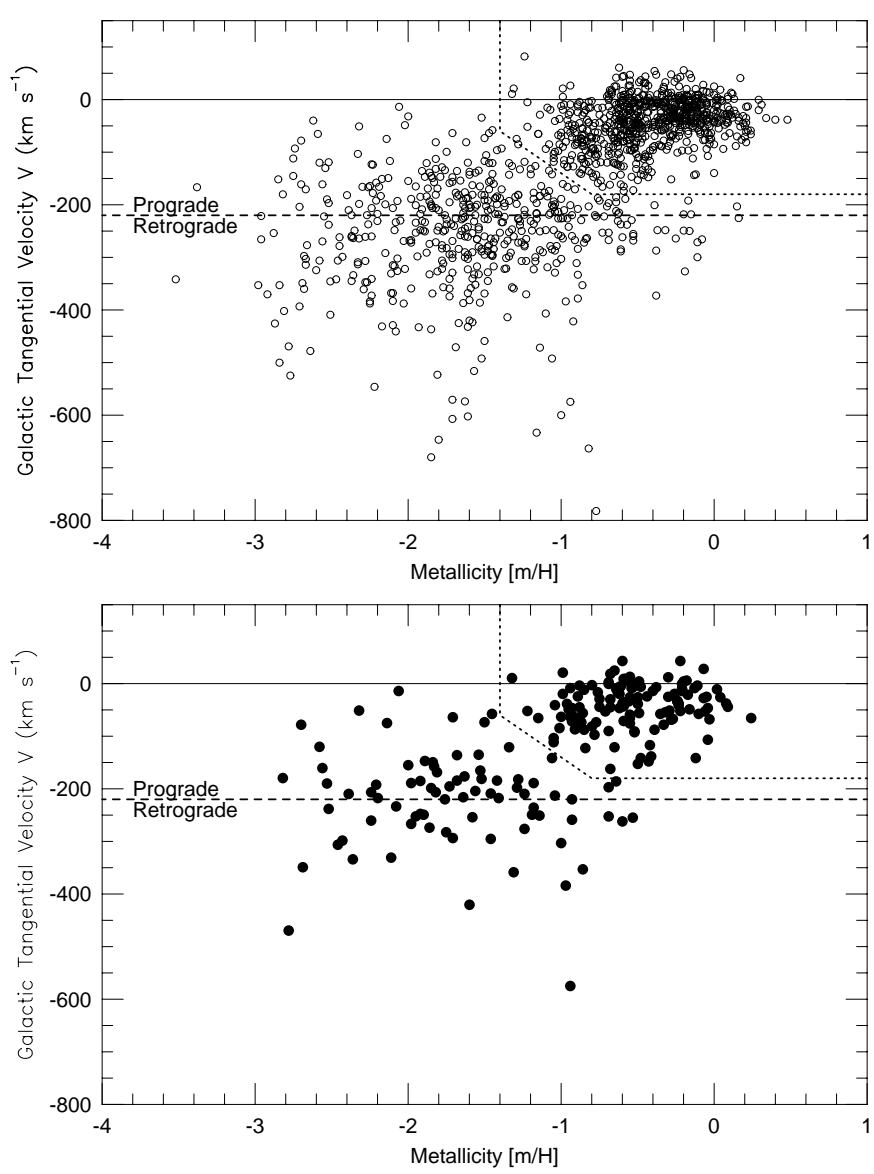

FIG. 7.-Galactic $V$ velocity vs. metallicity for constant stars (top) and spectroscopic binaries (bottom). The contour used to separate halo from disk stars is shown by a dotted line

distributions are the same for the halo and disk suggests that metallicity in general, and radiative opacities in particular, have little influence over the fragmentation process that leads to short-period binaries. Moreover, global differences in the dynamical state of the halo compared to the disk appear not to be important for this process.

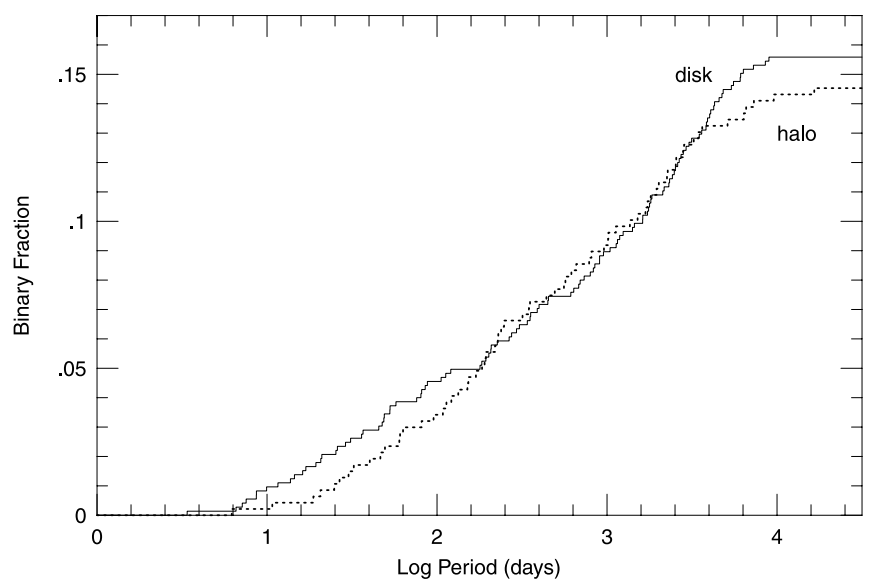

FIG. 8. - Cumulative period distributions for the disk (solid line) and halo binaries (dotted line).

\section{ECCENTRICITY VERSUS PERIOD}

In a landmark study of the frequency and orbital characteristics of the binaries found in a volume-limited sample of 164 solar-type stars, Duquennoy \& Mayor (1991) showed that the vast majority of their binaries had eccentric orbits. As had been previously noticed in other samples, the shortest period binaries all had circular orbits, which could be understood as the result of orbital circularization due to tidal mechanisms.

The plot of eccentricity versus log period for the 171 single-lined binaries from this paper (open circles) and the 34 double-lined binaries from Paper XV (filled circles) in Figure 9 shows the same general pattern as was found by Duquennoy \& Mayor (1991) for their sample of solar-type stars. All the binaries with periods shorter than about 10 days have orbits that are nearly circular, while only four binaries with periods longer than 30 days (G171-23, G250-43, G73-57, and G169-13) have nearly circular orbits. Perhaps these four systems formed with nearly circular orbits, or perhaps their orbits were circularized during the post-main-sequence evolution of the companion, when tidal circularization must have been much more effective, because giants have much larger convective zones than dwarfs. The minimum companion masses are $0.46,0.49$, 0.31 , and $0.17 M_{\odot}$, respectively, for these four binaries, which is not inconsistent with the companions all being white dwarfs with masses near $0.55 M_{\odot}$.

For periods longer than 30 days, the halo and disk populations of spectroscopic binaries have very similar eccentricity distributions. The mean eccentricity for the 62 such halo binaries is 0.414 with an rms dispersion of 0.218 and formal uncertainty in the mean value of \pm 0.028 . The corresponding result for the 95 such disk binaries is a mean eccentricity of $0.428 \pm 0.023$ and rms dispersion of 0.224 . Of course, these mean eccentricities are systematically too small because of the strong observational bias against detecting and solving binaries with highly eccentric orbits.

In Figure 10, we show the distribution of eccentricity versus log period for the metal-poor, high-velocity halo binaries defined by the contour plotted on Figure 7. The transition from circular to eccentric orbits for the halo appears to occur at a period of about 20 days, defined by G65-22 with a period of 18.74 days and G20-63 with a

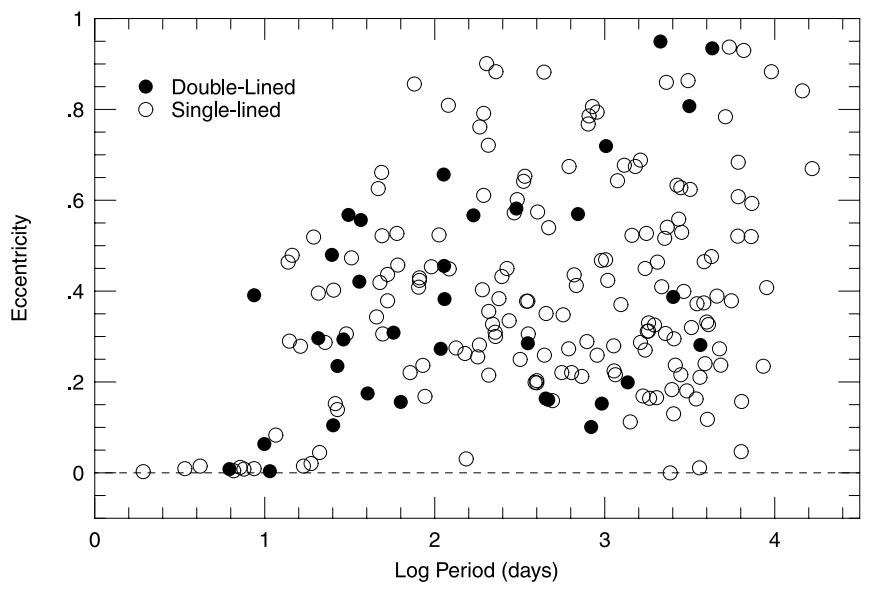

FIG. 9.-Eccentricity vs. log period for 171 single-lined binaries (open circles; this paper) and 34 double-lined binaries (filled circles; Paper XV). 


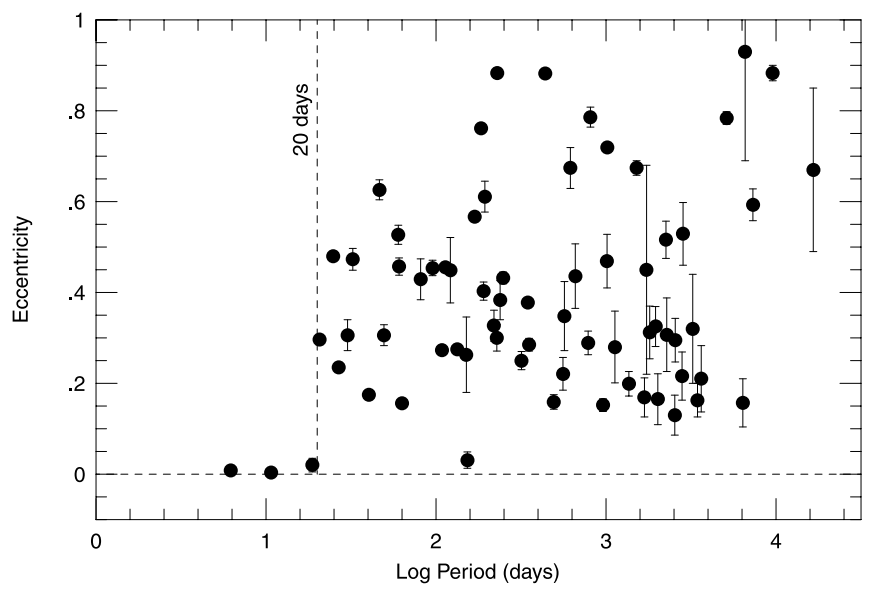

FIG. 10.-Same as Fig. 9, but for 61 halo binaries

period of 20.63 days. However, this result must be viewed with caution because of the woefully small number of binaries available in the critical transition region from 10 to 30 days and the difficulty of separating members of the halo and disk populations. For example, the binary G87-47 has a period of 13.7 days and an eccentricity of 0.464 . With a spectroscopic metallicity of $[\mathrm{m} / \mathrm{H}]=-1.32$, it lies near the limit of $[\mathrm{m} / \mathrm{H}]=-1.4$ that we chose somewhat arbitrarily as the dividing line between the halo and disk populations. Although its $V$ velocity is only $+11 \mathrm{~km} \mathrm{~s}^{-1}$, it has substantial $U$ and $W$ velocities of -112 and $63 \mathrm{~km} \mathrm{~s}^{-1}$, which suggest it is a member of the old thick disk or maybe even the halo. On the other hand, its metallicity is rather uncertain. Its Strömgren photometry leads to a metallicity of $[\mathrm{Fe} / \mathrm{H}]=-0.67$ (Schuster \& Nissen 1988), suggesting it belongs to the thick disk. Another intriguing piece of information is that Hipparcos found a substantial cosmic scatter in the astrometric solution for G87-47, $\epsilon=10.4$ mas. If this system is indeed a triple, then the distant third star might have induced the eccentricity that we observe for the inner spectroscopic binary (Mazeh \& Shaham 1979; Mazeh 1990).

\section{CONCLUSIONS}

We report 25,563 radial velocity measurements obtained with the CfA Digital Speedometers for 1359 single-lined stars in the full Carney-Latham sample of 1464 stars selected for high proper motion. The individual velocity errors range from about 0.5 to $1.5 \mathrm{~km} \mathrm{~s}^{-1}$, depending primarily on the metallicity of the star. Seven or more observations spanning more than 3000 days are available for nearly all the stars. In some cases, the time spanned by the observations reaches $20 \mathrm{yr}$. We have identified 188 singlelined binaries (not counting four triple systems that are single-lined but show two periods) in this sample and report orbital solutions for 171 .

We use metallicity and Galactic $V$ velocity to assign stars to the halo and disk populations. We find no obvious difference between the binary characteristics in the halo and disk, based on an analysis of 181 single-lined and double-lined (Paper XV) binaries with sufficient information. The observed binary frequency is the same, and the period distri- butions are consistent with the hypothesis that the two sets of binaries were drawn from the same parent population. Our period distributions are consistent with the speculation (Paper XIV) that a few of our halo binaries that initially had short periods have undergone mass transfer as the original primary evolved, resulting in orbital evolution to longer periods. Our comparison of the binaries in the halo and the disk may actually be valid for periods up to 10 or $20 \mathrm{yr}$, because our observing protocols did not include any biases that would favor one population over the other, except that our ability to detect low-amplitude spectroscopic binaries is poorer for more metal-poor stars. The fact that the period distributions are the same for the halo and disk suggests that metallicity in general, and radiative opacities in particular, have little influence over the fragmentation process that leads to short-period binaries.

All the binaries with periods shorter than 10 days have nearly circular orbits, while the binaries with periods longer than 20 days exhibit a wide range of eccentricities and a median value of about 0.37 . For the metal-poor, highvelocity halo binaries in our sample, the transition from circular to eccentric orbits appears to occur at about 20 days. However, this is not a strong conclusion because of the limited number of orbital solutions available in the critical range of periods from 10 to 30 days and the difficulty of assigning binaries unambiguously to the halo or disk. Nevertheless, this result supports the conclusion that tidal circularization on the main sequence is important for the oldest binaries in the Galaxy.

Finally, we comment on the growing evidence that the parent stars in systems harboring planets found by radial velocity searches tend to be more metal-rich than the Sun (e.g., Gonzalez et al. 2001). Truly metal-poor halo stars are rather rare, with a frequency of about 1 in 500 in the solar neighborhood. Only a handful of metal-poor stars have been targeted so far by the planet searchers. The absence of evidence for planets orbiting metal-poor stars is not evidence for their absence. If planets do indeed prove to be rare around metal-poor stars, then this would provide compelling evidence that stellar companions and planetary companions form and/or evolve by two different processes, because the frequency and orbital characteristics of stellar companions does not depend on metallicity. The metalpoor stars for which we do not detect velocity variations would make a good sample for testing the question of whether planets occur around metal-poor stars.

We dedicate this paper to the memory of Jim Peters, who obtained 3560 of the more than 31,000 spectra that have been accumulated for the Carney-Latham proper-motion sample. We will remember Jim as a loyal friend and an outstanding observer who could always be relied upon. We also thank Joe Caruso, Ed Horine, Joe Zajac, Perry Berlind, Ale Milone, Skip Schwartz, and Dick McCroskey for making many of the observations. We extend our sincerest thanks and appreciation to Bob Kurucz for his generous support of the effort to calculate new synthetic templates. We thank the referee for a very careful reading of the paper and for illuminating comments that have led to significant improvements. This work has been supported by the National Science Foundation through grants to the University of North Carolina and Bowling Green State University and by USIsrael Binational Science Foundation grants to Tel Aviv University. 
Abt, H. A. \& Levy, S. G. 1969, AJ, 74,908

Abt, H. A., \& Willmarth D. W. 1987, ApJ, 318, 786

Carney, B. W., Aguilar, L., Latham, D. W., \& Laird, J. B. 1990, AJ, 99, 201

Carney, B. W., Laird, J. B., Latham, D. W., \& Aguilar, L. 1996, AJ, 112, 668

Carney, B. W., Laird, J. B., Latham, D. W., \& Kurucz, R. L. 1987, AJ, 94, 1066

Carney, B. W., \& Latham, D. W. 1987, AJ, 93, 116

Carney, B. W., Latham, D. W., \& Laird, J. B. 1989, AJ, 97, 423 1990, AJ, 99, 572

Carney, B. W., Latham, D. W., Laird, J. B., \& Aguilar, L. 1994, AJ, 107, 2240 (Paper XII)

Carney, B. W., Latham, D. W., Laird, J. B., Grant, C. E., \& Morse, J. A. 2001, AJ, 122, 3419 (Paper XIV)

Carney, B. W., Latham, D. W., Stefanik, R. P., Morse, J. A., \& Laird, J. B. 2002 , in preparation

Carquillat, J. M., Nadal, R., Ginestet, N., \& Pedoussaut, A. 1983, A\&AS, 54,187

Crampton, D., \& Hartwick, F. D. 1972, AJ, 77, 590

Duquennoy, Ä., \& Mayor, M. 1991, A\&A, 248, 485

Giclas, H. L., Burnham, R., Jr., \& Thomas, N. G. 1971, Lowell Proper Motion Survey, Northern Hemisphere (Flagstaff: Lowell Obs.) .1978, Lowell Obs. Bull., 8, 89

Goldberg, D., Mazeh, T., Latham, D. W., Stefanik, R. P., Carney, B. W., \& Laird, J. B. 2002, AJ, 124, 1132

Gonzalez, G., Laws, C., Tyagi, S., \& Reddy, B. E. 2001, AJ, 121, 432

Griffin, R. F. 1981, J. Astrophys. Astron., 2, 309

Gunn, J. E., \& Griffin, R. F. 1979, AJ, 84, 752

Jaschek, C., \& Jaschek, M. 1957, Z. Astrophys.

Jasniewicz, G., \& Mayor, M. 1986, A\&A, 170, 55 . 1988, A\&A, 203, 329

Johnson, H. M. \& Mayor, M. 1986, ApJ, 310, 354

Kurtz, M. J., \& Mink, D. J. 1998, PASP, 110, 934

Kurucz, R. L., Furenlid, I., Brault, J., \& Testerman, L. 1984, Solar Flux Atlas from 296 to $1300 \mathrm{~nm}$ (Sunspot: Natl. Solar Obs.)

Laird, J. B., Rupen, M. P., Carney, B. W., \& Latham, D. W. 1988, AJ, 96, 1908

Latham, D. W. 1985, in IAU Colloq. 88, Stellar Radial Velocities, ed.

A. G. D. Philip \& D. W. Latham (Schenectady: L. Davis), 21

\section{REFRENCES}

Latham, D. W. 1992, in ASP Conf. Ser. 32, Complementary Approaches to Binary and Multiple Star Research, ed. H. McAlister \& W. Hartkopf (IAU Colloq. 135) (San Francisco: ASP), 110

Latham, D. W., Mazeh, T., Carney, B. W., McCroskey, R. E., Stefanik, R. P., \& Davis, R. J. 1988, AJ, 96, 567 (Paper VI)

Latham, D. W., et al. 1992, AJ, 104, 774 (Paper XI)

Latham, D. W., Mazeh, T., Stefanik, R. P., Mayor, M., \& Burki, G. 1989, Nature, 339, 38

Marcy, G. W., Butler, R. P., Vogt, S. S., Fischer, D., \& Liu, M. C. 1999, ApJ, 520, 239

Mathieu, R. D., Duquennoy, A., Latham, D. W., Mayor, M., Mazeh, T., \& Mermilliod, J.C. 1992, in Binaries as Tracers of Stellar Formation, ed. A. Duquennoy \& M. Mayor (Cambridge: Cambridge Univ. Press), 278

Mayor, M., \& Turon, C. 1982, A\&A, 110, 241

Mazeh, T. 1990, AJ, 99, 675

Mazeh, T., Krymolowski, Y., \& Latham, D. W. 1993, MNRAS, 263, 775

Mazeh, T., Latham, D. W., \& Stefanik, R. P. 1996, ApJ, 466, 415

Mazeh, T., Martín, E. L., Goldberg, D., \& Smith, H. A. 1997, MNRAS, 284,341

Mazeh, T., \& Shaham, J. 1979, A\&A, 77, 145

Morse, J. A., \& Kurucz, R. L. 2002, in preparation

Nordström, B., Latham, D. W., Morse, J. A., Milone, A. A. E., Kurucz, R. L., Andersen, J., \& Stefanik, R. P. 1994, A\&A, 287, 338

Press, W. H., Teukolsky, S. A., Vetterling, W. T., \& Flannery, B. P. 1992, Numerical Recipes in Fortran (2d ed; Cambridge: Cambridge Univ. Press)

Schuster, W. J., \& Nissen, P. E. 1988, A\&AS, 73, 225

Stefanik, R. P., Latham, D. W., \& Torres, G. 1999, in ASP Conf. Ser. 185 , Precise Stellar Radial Velocities, ed. J. B. Hearnshaw \& C. D. Scarfe (IAU Colloq. 170) (San Francisco: ASP), 354

Stockton, R. A., \& Fekel, F. C. 1992, MNRAS, 256, 575

Stryker, L. L., Hesser, J. E., Hill, G., Garlick, G. S., \& O'Keefe, L. M. 1985, PASP, 97, 247

Tokovinin, A. A. 1992, A\&A, 256, 121

Tokovinin, A. A., Duquennoy, A., Halbwachs, J.-L., \& Mayor, M. 1994 A\&A, 282, 831

Torres, G. 1991, Ph.D. thesis, Univ. Córdoba

Zucker, S., \& Mazeh, T. 1994, ApJ, 420, 806

Zucker, S., Torres, G., \& Mazeh, T. 1995, ApJ, 452, 863 